\title{
Density profiles around nanoparticles and distant perturbations
}

E. Eisenriegler

Citation: The Journal of Chemical Physics 130, 134902 (2009); doi: 10.1063/1.3091939

View online: https://doi.org/10.1063/1.3091939

View Table of Contents: http://aip.scitation.org/toc/jcp/130/13

Published by the American Institute of Physics

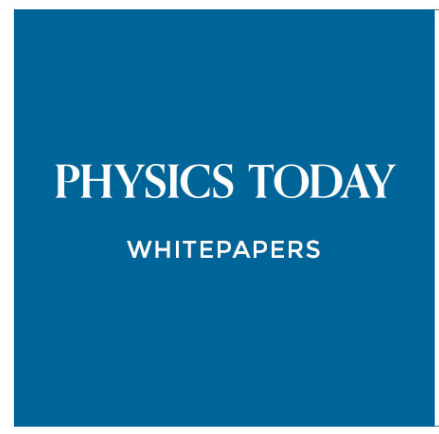
systems can do 


\title{
Density profiles around nanoparticles and distant perturbations
}

\author{
E. Eisenriegler ${ }^{\mathrm{a})}$ \\ Institut für Festkörperforschung, Forschungszentrum Jülich, D-52425 Jülich, Germany
}

(Received 23 October 2008; accepted 10 February 2009; published online 1 April 2009)

\begin{abstract}
We investigate how density profiles around a spherical nanoparticle in a critical solvent are affected by distant perturbations such as a wall or other particles. Using a new type of "fusion expansion," we evaluate the isotropic and anisotropic changes in the densities at distances $r$ from the sphere center which are of the order of the sphere radius $R$, with both $R$ and $r$ much smaller than the correlation length and the distance between the sphere and the perturbations. Our results bridge the gap between the changes in the region $0<r-R \ll R$ close to the sphere surface that determine the local pressure and force on the spherical particle and in the more distant region $R \ll r$ where the "small sphere expansion" applies. Applications to particles in critical binary liquid mixtures and in solutions of long, flexible nonadsorbing polymers are considered. (C) 2009 American Institute of Physics. [DOI: 10.1063/1.3091939]
\end{abstract}

\section{INTRODUCTION}

In a critical system the correlation length $\xi$ is large and local perturbations lead to long ranged effects. For example, two colloidal particles immersed in a critical solvent experience a long-ranged interaction. ${ }^{1}$ Intimately related is the change or correction of density profiles near one particle due to the presence of the other distant particle.

Fisher and de Gennes ${ }^{2}$ investigated the correction of the critical adsorption profile near a planar wall due to another wall. The parallel wall geometry is closely related to particles that are much larger than the surface-to-surface separation between them.

Here we consider a spherical particle $S$ centered at $\mathbf{r}_{c}$ with a radius $R$ that is much smaller than both $\xi$ and the closest separation $\mathcal{D}$ of $\mathbf{r}_{c}$ from the distant particle or wall, a situation first addressed by de Gennes ${ }^{3,4}$ and shown in Fig. 1, and we evaluate the corrections of its density profiles at distances $r$ from $\mathbf{r}_{c}$ that are of the order of $R$. The results are detail independent, i.e., "universal" if $R$ and the distance $r-R$ from the surface of $S$ are large on a microscopic scale. We explicitly determine the leading corrections that are isotropic and anisotropic about $\mathbf{r}_{c}$ and relate the latter to the force on the particle $S$. In the following we refer to $S$, with mesoscopic radius $R$ much smaller than $\mathcal{D}$ and $\xi$, as a "nanoparticle."

For density profiles near a planar wall, the corrections and their relation ${ }^{5-8}$ to the force ${ }^{2}$ on the wall are conveniently investigated by using an expansion for the density operator in terms of boundary operators. ${ }^{9}$ In the nanoparticle case we view the combination of the density operator and the nearby particle $S$ as a small object and expand it in terms of bulk operators ${ }^{10}$ and their derivatives. Besides density profile corrections, we also discuss correlation functions of densities, where one density is close to the sphere $S$.

With applications to critical adsorption of binary liquid mixtures ${ }^{1,3,11,12}$ and polymer depletion ${ }^{4,13}$ in mind, we study

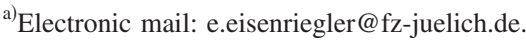

spheres with both "normal" and "ordinary" surfaces which break and conserve the order parameter symmetry, ${ }^{9}$ respectively, and we consider order parameter and energy densities $\phi$ and $\epsilon$. For simplicity, we assume that the bulk of the embedding critical system is in the nonordered state with conserved order parameter symmetry so that $\langle\phi\rangle_{\text {bulk }}$ vanishes.

\section{DENSITY CORRECTIONS DUE TO DISTANT PERTURBATIONS}

Since $R$ and $r$ are much smaller than other mesoscopic lengths, one may "fuse" the density operator $\psi=\phi$ or $\epsilon$ at $\mathbf{r}_{c}+\mathbf{r}$ with the sphere $S$ centered at $\mathbf{r}_{c}$ into a pointlike object located at $\mathbf{r}_{c}$, which can be represented by a series of operators and their derivatives. Denoting the surface universality class (normal or ordinary) of $S$ by $a$, the lowest order terms of this series are given by

$$
\begin{aligned}
\mathcal{F}^{(\psi, a)}\left(R, \mathbf{r} ; \mathbf{r}_{c}\right)= & \sum_{\chi=\phi, \epsilon}\left\{\mathcal{A}_{\chi}^{(\psi, a)}(R, r)\right. \\
& \left.+\widetilde{\mathcal{A}}_{\chi}^{(\psi, a)}(R, r) \mathbf{r} \frac{\partial}{\partial \mathbf{r}_{c}}\right\} \chi\left(\mathbf{r}_{c}\right) / \sqrt{B_{\chi}}
\end{aligned}
$$

with universal amplitude functions

$$
\left(\mathcal{A}_{\chi}^{(\psi, a)}(R, r), \widetilde{\mathcal{A}}_{\chi}^{(\psi, a)}(R, r)\right)=R^{x} \chi^{-x} \psi y^{-x} \psi\left(1, \frac{1}{x_{\chi}} y \frac{\mathrm{d}}{\mathrm{d} y}\right) X_{\psi, \chi}^{(a)}(y)
$$

and

$$
y=\left(\frac{r}{R}\right)^{2}-1
$$

The functions $X$ in Eq. (2.2) follow from the two-point cumulants $\langle\psi \cdot \chi\rangle \equiv\langle\psi \chi\rangle-\langle\psi\rangle\langle\chi\rangle$ in the half space (hs) with surface class $a$, at the critical point, where ${ }^{14}$ 


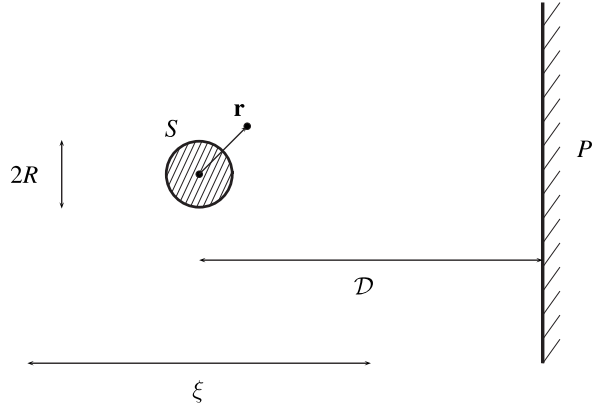

FIG. 1. Spherical particle $S$ with center at a distance $\mathcal{D}$ from a planar wall $P$. We consider the case in which the particle radius $R$ is much smaller than $\mathcal{D}$ and the correlation length $\xi$ and study the density profiles induced by $S$ and $P$ at distances $r=|\mathbf{r}|$ from the particle center which are of the order of $R$, i.e., for $R, r \ll \mathcal{D}, \xi$.

$$
\begin{aligned}
\left\langle\psi\left(\mathbf{r}_{1}\right) \cdot \chi\left(\mathbf{r}_{2}\right)\right\rangle_{\mathrm{hs}}^{(a)}= & \sqrt{B_{\psi} B_{\chi}}\left(2 r_{1 \perp}\right)^{-x} \psi\left(2 r_{2 \perp}\right)^{-x} \chi \\
& \times X_{\psi, \chi}^{(a)}\left(\frac{4 r_{1 \perp} r_{2 \perp}}{\left|\mathbf{r}_{1}-\mathbf{r}_{2}\right|^{2}}\right) .
\end{aligned}
$$

Here $r_{1 \perp}$ is the component of $\mathbf{r}_{1}$ perpendicular to the planar boundary of the half space and $x_{\psi}$ and $B_{\psi}$ denote the scaling dimensions of the operators $\psi$ and the nonuniversal amplitudes that appear in the corresponding bulk functions $\left\langle\psi\left(\mathbf{r}_{1}\right) \psi\left(\mathbf{r}_{2}\right)\right\rangle_{\text {bulk }}=B_{\psi}\left|\mathbf{r}_{1}-\mathbf{r}_{2}\right|^{-2 x_{\psi}}$. The mixed function $X_{\phi, \epsilon}^{(a)}(y)=X_{\epsilon, \phi}^{(a)}(y)$ only appears for the symmetry breaking surface $a=$ normal and the term for $\chi \neq \psi$ in Eq. (2.1) vanishes for an ordinary surface.

Besides the anisotropic term in Eq. (2.1), which is of order $x_{\chi}-x_{\psi}+1$ in the small lengths $R$ and $r$ and is odd in $\mathbf{r}$, there are also anisotropic terms in the fusion which are even in $\mathbf{r}$ such as $\sum_{k, l} r_{k} r_{l}\left(\partial^{2} / \partial r_{c k} \partial r_{c l}\right) \chi\left(\mathbf{r}_{c}\right)$ and $\sum_{k, l} r_{k} r_{l} T_{k l}\left(\mathbf{r}_{c}\right)$. Here $T_{k l}$ is the stress tensor ${ }^{15}$ whose scaling dimension equals the spatial dimension $d$. Since their amplitude functions contain prefactors $R^{x^{-}-x_{\psi}}$ and $R^{d-2-x_{\psi}}$, respectively, they are of order $x_{\chi}-x_{\psi}+2$ and $d-x_{\psi}$ in the small lengths. Thus, they lead, in general, to smaller contributions than the odd anisotropy and except in Appendix A 2 we shall disregard them.

We now consider two applications. (i) The correction

$$
\left\langle\psi\left(\mathbf{r}_{c}+\mathbf{r}\right)\right\rangle_{S, P}-\left\langle\psi\left(\mathbf{r}_{c}+\mathbf{r}\right)\right\rangle_{S} \equiv \delta_{P}\left\langle\psi\left(\mathbf{r}_{c}+\mathbf{r}\right)\right\rangle_{S}
$$

of the density $\langle\psi\rangle_{S}$ due to a wall or particle $P$ with closest separation $\mathcal{D}$ from $\mathbf{r}_{c}$ tends to

$$
\begin{aligned}
\delta_{P}\left\langle\psi\left(\mathbf{r}_{c}+\mathbf{r}\right)\right\rangle_{S} / \sqrt{B_{\psi}} & \rightarrow\left\langle\mathcal{F}^{(\psi, a)}\right\rangle_{P}-\left\langle\mathcal{F}^{(\psi, a)}\right\rangle_{\text {bulk }} \\
= & \sum_{\chi=\phi, \epsilon}\left\{\mathcal{A}_{\chi}^{(\psi, a)}+\widetilde{\mathcal{A}}_{\chi}^{(\psi, a)} \mathbf{r} \frac{\partial}{\partial \mathbf{r}_{c}}\right\} \\
& \times \delta_{P}\left\langle\chi\left(\mathbf{r}_{c}\right)\right\rangle_{\text {bulk }} / \sqrt{B_{\chi}}
\end{aligned}
$$

as $\mathcal{D}$ and $\xi$ become much larger than $R$ and $r$. Here $\delta_{P}\langle\chi\rangle_{\text {bulk }} \equiv\langle\chi\rangle_{P}-\langle\chi\rangle_{\text {bulk }}$ contains averages where $S$ is absent. (ii) For multipoint averages with operators $\mathcal{O}_{i}$ at points in the space outside $S$ and $P$, a similar reduction, ${ }^{10}$

$$
\begin{aligned}
{\left[\left\langle\mathcal{O}_{1}\left(\mathbf{r}_{1}\right) \ldots \mathcal{O}_{n}\left(\mathbf{r}_{n}\right) \psi\left(\mathbf{r}_{c}+\mathbf{r}\right)\right\rangle_{S, P}\right.} \\
\left.-\left\langle\mathcal{O}_{1}\left(\mathbf{r}_{1}\right) \ldots \mathcal{O}_{n}\left(\mathbf{r}_{n}\right)\right\rangle_{S, P}\left\langle\psi\left(\mathbf{r}_{c}+\mathbf{r}\right)\right\rangle_{S, P}\right] / \sqrt{B_{\psi}} \\
\quad \rightarrow\left\langle\mathcal{O}_{1}\left(\mathbf{r}_{1}\right) \ldots \mathcal{O}_{n}\left(\mathbf{r}_{n}\right) \mathcal{F}^{(\psi, a)}\right\rangle_{P} \\
\quad-\left\langle\mathcal{O}_{1}\left(\mathbf{r}_{1}\right) \ldots \mathcal{O}_{n}\left(\mathbf{r}_{n}\right)\right\rangle_{P}\left\langle\mathcal{F}^{(\psi, a)}\right\rangle_{P},
\end{aligned}
$$

applies as $\mathcal{D}, \xi$, and $\left|\mathbf{r}_{1}-\mathbf{r}_{c}\right|, \ldots,\left|\mathbf{r}_{n}-\mathbf{r}_{c}\right|$ become much larger than $R$ and $r$. This relation is nontrivial even in the absence of $P$, where \langle\rangle$_{P}=\langle\rangle_{\text {bulk. }}$. On the right-hand sides of Eqs. (2.6) and (2.7) the derivatives $\partial / \partial \mathbf{r}_{c}$ of $\left\langle\chi\left(\mathbf{r}_{c}\right)\right\rangle_{P}$ and $\left\langle\mathcal{O}_{1}\left(\mathbf{r}_{1}\right) \ldots \mathcal{O}_{n}\left(\mathbf{r}_{n}\right) \chi\left(\mathbf{r}_{c}\right)\right\rangle_{P}$ are evaluated at fixed positions of the walls or particles $P$ and at fixed $\mathbf{r}_{1}, \ldots, \mathbf{r}_{n}$.

The above case of both $R$ and $r$ small should be distinguished from the case where $R$ is the only small mesoscopic length, so that the "small sphere expansion" applies ${ }^{16}$ to $S$. For the Boltzmann weight of a sphere $S$ with surface class $a$, radius $R$, and center at $\mathbf{r}_{c}$, this expansion reads

$$
\begin{aligned}
& \exp \left[-\Delta \mathcal{H}_{a}\left(R ; \mathbf{r}_{c}\right)\right] \propto 1+\sigma, \quad \sigma=\sigma_{\phi}+\sigma_{\epsilon}+\ldots, \\
& \sigma_{\psi}=\left(A_{\psi}^{(a)} / B_{\psi}\right) R^{x} \psi \psi\left(\mathbf{r}_{c}\right),
\end{aligned}
$$

see Refs. 17-19. Here $A_{\psi}^{(a)}$ is the amplitude of the profile $\left\langle\psi\left(r_{\perp}\right)\right\rangle_{\mathrm{hs}}^{(a)}=A_{\psi}^{(a)}\left(2 r_{\perp}\right)^{-x_{\psi}}$ in the half space at the critical point and with surface class $a$. The amplitude $A_{\phi}$ vanishes in the ordinary case of free surface spins and is positive (negative) for a normal surface $+(-)$ with surface spins fixed in the positive (negative) spin direction. Here we consider the Ising model describing critical adsorption of binary fluid mixtures. ${ }^{9,12}$ The amplitude $A_{\epsilon}$ is positive for an ordinary and negative for a normal surface, since ordinary and normal surfaces of free and fixed spins induce disorder and order, respectively, of the spins in the half space.

Using the small sphere expansion for a small distant spherical particle $P$ converts Eq. (2.6) to the form of Eq. (2.7), with $n=1$ and $P$ absent.

For $a=$ normal the leading contribution from the distant perturbations to the quantities in Eq. (2.6) or (2.7) is provided by $\chi=\phi$ in the sum of Eq. (2.1) if $\langle\phi\rangle_{P}$ or $\left\langle\mathcal{O}_{1} \ldots \mathcal{O}_{n} \phi\right\rangle_{P}$ are nonvanishing and by $\chi=\epsilon$ otherwise. For $\psi=\phi$ and $\langle\phi\rangle_{P}=0$ and for $\psi=\epsilon$ and $\langle\phi\rangle_{P} \neq 0$ the nondiagonal scaling function $X_{\phi, \epsilon}^{(\text {normal })}$ provides the leading contribution in Eq. (2.6).

\section{A. Simple examples}

Here we recall some known results and show that they obey the fusion relations (2.1)-(2.7).

First we check Eq. (2.7) for the two-point cumulant outside $S$ with $P$ absent and the bulk right at the critical point. Assuming that $\mathcal{O}_{1}$ is equal to $\phi$ or $\epsilon$, the form

$$
\begin{aligned}
\left\langle\mathcal{O}_{1}\left(\mathbf{r}_{1}\right) \cdot \psi\left(\mathbf{r}_{c}+\mathbf{r}\right)\right\rangle_{S} / \sqrt{B_{\psi}} \\
=\sqrt{B_{\mathcal{O}_{1}}}\left(\frac{R}{\left|\mathbf{r}_{1}-\mathbf{r}_{c}\right|^{2}-R^{2}}\right)^{x_{1}}(R y)^{-x_{\psi}} \\
\quad \times X_{\psi, \mathcal{O}_{1}}^{(a)}\left(y \frac{\left|\mathbf{r}_{1}-\mathbf{r}_{c}\right|^{2}-R^{2}}{\left|\mathbf{r}_{1}-\mathbf{r}_{c}-\mathbf{r}\right|^{2}}\right)
\end{aligned}
$$


of the left-hand side of Eq. (2.7) follows ${ }^{20}$ from a conformal transformation from the two-point cumulant in the half space given in Eq. (2.4). Since $\langle\phi \cdot \epsilon\rangle_{\text {bulk }}$ vanishes, only $\chi=\mathcal{O}_{1}$ contributes to the right-hand side of (2.7), which is given by

$$
\begin{aligned}
& \left\langle\mathcal{O}_{1}\left(\mathbf{r}_{1}\right) \cdot \mathcal{F}^{(\psi, a)}\right\rangle_{\text {bulk }} \\
& \quad=\frac{\sqrt{B_{\mathcal{O}_{1}}}}{\left|\mathbf{r}_{1}-\mathbf{r}_{c}\right|^{2 x_{1}}}\left\{\mathcal{A}_{\mathcal{O}_{1}}^{(\psi, a)}+2 \frac{\mathbf{r}\left(\mathbf{r}_{1}-\mathbf{r}_{c}\right)}{\left|\mathbf{r}_{1}-\mathbf{r}_{c}\right|^{2}} x_{1} \widetilde{\mathcal{A}}_{\mathcal{O}_{1}}^{(\psi, a)}\right\}
\end{aligned}
$$

and contains the leading and next to leading orders $\propto\left|\mathbf{r}_{1}-\mathbf{r}_{c}\right|^{-2 x_{1}}$ and $\propto\left|\mathbf{r}_{1}-\mathbf{r}_{c}\right|^{-2 x_{1}-1}$ of Eq. (2.9). Here $x_{1}$ is the scaling dimension of $\mathcal{O}_{1}$.

(ii) To show that the fusion (2.1)-(2.4) of $\psi$ with $S$ also applies away from the critical point, we consider Eq. (2.7) for the order-parameter two-point function in the Gaussian model of Appendix A 1 outside a sphere $S$ with an ordinary surface and center at $\mathbf{r}_{c}=\mathbf{0}$. This has the form ${ }^{21}$

$$
\begin{aligned}
\left\langle\phi\left(\mathbf{r}_{1}\right) \phi(\mathbf{r})\right\rangle_{S} / B_{\phi}= & \sum_{l=0}^{\infty} r^{-\alpha} \gamma_{l}(t, R, r) 2(\alpha+l) \\
& \times C_{l}^{(\alpha)}(\cos \vartheta) r_{1}^{-\alpha} K_{\alpha+l}\left(t^{1 / 2} r_{1}\right)
\end{aligned}
$$

for $r<r_{1}$. Here $\alpha=(d-2) / 2$ equals $x_{\phi}$ and depends on the spatial dimension $d$,

$$
\gamma_{l}=I_{\alpha+l}\left(t^{1 / 2} r\right)-\left[I_{\alpha+l}\left(t^{1 / 2} R\right) / K_{\alpha+l}\left(t^{1 / 2} R\right)\right] K_{\alpha+l}\left(t^{1 / 2} r\right),
$$

where $t^{1 / 2}$ is proportional to the inverse correlation length $1 / \xi, \vartheta$ is the angle between $\mathbf{r}$ and $\mathbf{r}_{1}$, and $C_{l}^{(\alpha)}$, $I_{\nu}$, and $K_{\nu}$ are Gegenbauer polynomials and modified Bessel functions. Since both the bulk and the boundary condition preserve the symmetry of the order parameter, $\langle\phi\rangle_{S}$ vanishes and $\langle\phi \phi\rangle_{S}=\langle\phi \cdot \phi\rangle_{S}$. For $R, r$ $\ll r_{1}, \xi$

$\gamma_{l} \rightarrow \frac{\left(t^{1 / 2} r / 2\right)^{\alpha+l}}{\Gamma(\alpha+l+1)}\left[1-\left(\frac{R}{r}\right)^{2(\alpha+l)}\right]$,

and the leading isotropic and anisotropic r-dependence is determined by the term $l=0$ and $l$ $=1$, respectively. This yields

$$
\begin{aligned}
\left\langle\phi\left(\mathbf{r}_{1}\right) \phi(\mathbf{r})\right\rangle_{S} / B_{\phi} \rightarrow & \left\{\mathcal{A}_{\phi}^{(\phi, \text { ord })} K_{\alpha}\left(t^{1 / 2} r_{1}\right)\right. \\
& \left.+\widetilde{\mathcal{A}}_{\phi}^{(\phi, \text { ord })} t^{1 / 2} r(\cos \vartheta) K_{\alpha+1}\left(t^{1 / 2} r_{1}\right)\right\} \\
& \times\left(t^{1 / 2} / r_{1}\right)^{\alpha} 2^{1-\alpha} / \Gamma(\alpha) \\
\equiv & \left\{\mathcal{A}_{\phi}^{(\phi, \text { ord })}+\widetilde{\mathcal{A}}_{\phi}^{(\phi, \text { ord })} \mathbf{r} \frac{\partial}{\partial \mathbf{r}_{c}}\right\} \\
& \times\left\langle\phi\left(\mathbf{r}_{1}\right) \phi\left(\mathbf{r}_{c}\right)\right\rangle_{\mathrm{bulk}} /\left.B_{\phi}\right|_{\mathbf{r}_{c}=0}, \quad(2.14)
\end{aligned}
$$

with the amplitudes

$$
\mathcal{A}_{\phi}^{(\phi, \text { ord })}=1-(R / r)^{2 \alpha}, \quad \widetilde{\mathcal{A}}_{\phi}^{(\phi, \text { ord })}=1-(R / r)^{2(\alpha+1)} .
$$

Since these amplitudes also follow via Eq. (2.2) from the half-space scaling function

$X_{\phi, \phi}^{(\text {ord })}(y)=y^{\alpha}\left[1-(1+y)^{-\alpha}\right]$

of the Gaussian model at the critical point, Eq. (2.14) is consistent with the fusion equation (2.7) for $n=1$ with $\mathcal{O}_{1}=\psi=\phi$ in the absence of the perturbation $P$. Here the form (2.1) of $\mathcal{F}^{(\phi, \text { ord })}$ and the vanishing of $\mathcal{A}_{\phi}^{(\epsilon, \text { ord })}$ and $\widetilde{\mathcal{A}}_{\phi}^{(\epsilon, \text { ord })}$ have been used.

(iii) Next consider the two-point cumulant of the energy density $\epsilon \propto-\phi^{2}$ outside the Dirichlet sphere in the Gaussian model ${ }^{22}$

$$
\begin{aligned}
\left\langle\epsilon\left(\mathbf{r}_{1}\right) \cdot \epsilon(\mathbf{r})\right\rangle_{S} / B_{\epsilon} & =\left\langle\phi^{2}\left(\mathbf{r}_{1}\right) \cdot \phi^{2}(\mathbf{r})\right\rangle_{S} / B_{\phi^{2}} \\
& =\left(\left\langle\phi\left(\mathbf{r}_{1}\right) \phi(\mathbf{r})\right\rangle_{S} / B_{\phi}\right)^{2},
\end{aligned}
$$

which is simply the square of the expression in Eq. (2.11). Its leading isotropic and anisotropic $\mathbf{r}$-behavior follows from the square of Eq. (2.14), on discarding the square of the derivative term, so that

$$
\begin{aligned}
\left\langle\epsilon\left(\mathbf{r}_{1}\right) \cdot \epsilon(\mathbf{r})\right\rangle_{S} / B_{\epsilon} \rightarrow & \left\{\mathcal{A}_{\epsilon}^{(\epsilon, \text { ord })}+\widetilde{\mathcal{A}}_{\epsilon}^{(\epsilon \text {,ord })} \mathbf{r} \frac{\partial}{\partial \mathbf{r}_{c}}\right\} \\
& \times\left\langle\epsilon\left(\mathbf{r}_{1}\right) \cdot \epsilon\left(\mathbf{r}_{c}\right)\right\rangle_{\text {bulk }} / B_{\epsilon} \mid \mathbf{r}_{c}=0
\end{aligned}
$$

where

$\mathcal{A}_{\epsilon}^{(\epsilon, \text { ord })}=\left(\mathcal{A}_{\phi}^{(\phi, \text { ord })}\right)^{2}, \quad \widetilde{\mathcal{A}}_{\epsilon}^{(\epsilon, \text { ord })}=\mathcal{A}_{\phi}^{(\phi, \text { ord })} \tilde{\mathcal{A}}_{\phi}^{(\phi, \text { ord })}$.

The amplitudes $\mathcal{A}_{\epsilon}^{(\epsilon, \text { ord })}$ and $\tilde{\mathcal{A}}_{\epsilon}^{(\epsilon, \text { ord })}$ are also obtained via Eq. (2.2) from the scaling function

$X_{\epsilon, \epsilon}^{\text {(ord) }}(y)=\left[X_{\phi, \phi}^{\text {(ord) }}(y)\right]^{2}$

at the critical point, and Eq. (2.18) is consistent with Eq. (2.7).

(iv) For a weak perturbation $P$ such as a defect plane, where

$\delta_{P}\langle\psi\rangle_{S}=H_{\mathcal{O}_{1}} \int \mathrm{d}^{d-1} \mathbf{r}_{1 \|}\left\langle\mathcal{O}_{1}\left(\mathbf{r}_{1}\right) \cdot \psi\right\rangle_{S}$

and

$\delta_{P}\langle\chi\rangle_{\text {bulk }}=H_{\mathcal{O}_{1}} \int \mathrm{d}^{d-1} \mathbf{r}_{1 \|}\left\langle\mathcal{O}_{1}\left(\mathbf{r}_{1}\right) \cdot \chi\right\rangle_{\text {bulk }}$

to first order in the weak defect strength $H_{\mathcal{O}_{1}}$. For $\mathcal{O}_{1}=\phi$ the perturbation induces local order $\delta_{P}\langle\phi\rangle_{\text {bulk }}$ $\neq 0$ in the nonordered bulk $\langle\phi\rangle_{\text {bulk }}=0$, and for $\mathcal{O}_{1}=\epsilon$ and $H_{\epsilon}>0$ it increases the energy locally since $\delta_{P}\langle\epsilon\rangle_{\text {bulk }}>0$. In the framework of the Ising model, the two types of defect planes are weak versions of a normal and an ordinary planar surface of fixed and free spins, respectively. For the defect planes the form 
(2.6) of the density corrections follows from the relations (2.7) with $n=1$, considered above, by simple integration over $\mathbf{r}_{1 \|}$. A derivation of Eq. (2.6) for strong perturbations $P$ will be given in Sec. IV.

(v) Examples of Eq. (2.7) for $n>1$ and for nonvanishing perturbations $P$ in the Gaussian and $d=2$ Ising models are considered in Appendix A.

\section{CORRECTIONS CLOSE TO AND FAR FROM THE PARTICLE SURFACE}

The expression (2.6) or (2.7) for densities at a distance $r$ from the sphere center are valid for $r$ of the order of $R$, including the limits $|r-R| \ll R$ and $R \ll r$, i.e., close to and far from the sphere surface, as long as $r$ and $R$ are much smaller than $\xi$ and the distances to the perturbations. In this section we discuss the behavior in the two limits which are determined by "boundary operator expansions" and small sphere expansions, ${ }^{17}$ respectively.

\section{A. Corrections close to the surface and the force onto the particle}

In the parallel plate geometry of Fisher and de Gennes the form of the distant wall correction ${ }^{2}$ of the density profiles and its relation ${ }^{5-8}$ to the force between plates only apply to the combinations

$$
(\psi, a)=(\epsilon, \text { ord }),(\phi, \pm),(\epsilon, \pm)
$$

of the density $\psi$ and the surface class $a$ of the close wall. Here we have used the notation introduced below Eq. (2.8). This can be understood in terms of a boundary operator expansion of $\psi$ near a planar wall of class $a,{ }^{6-9}$ since for Eq. (3.1) the leading boundary operator is the diagonal component normal to the surface $T_{n n}$ of the stress tensor and since its surface-integrated thermal average is related to the force. This also applies to an operator expansion close to the curved boundary of $S$. Here we use Eq. (2.6) to evaluate the correction of $\langle\psi\rangle$ close to the surface of the nanosphere for the combinations (3.1) and relate its surface-integrated anisotropic part to the force $\mathbf{f}$ that the perturbation $P$ exerts onto the sphere $S$. The latter is obtained as the derivative $-\partial / \partial \mathbf{r}_{C}$ of the free energy $-k_{B} T \ln \left\langle\exp \left(-\Delta \mathcal{H}_{a}\right)\right\rangle_{P}$ following from the small sphere expansion (2.8) with the result ${ }^{17}$

$$
\frac{\mathbf{f}}{k_{B} T}=\frac{A_{\chi}^{(a)}}{B_{\chi}} R^{x} \chi \frac{\partial}{\partial \mathbf{r}_{c}}\left\langle\chi\left(\mathbf{r}_{c}\right)\right\rangle_{P}
$$

to leading order in $R$. Here $\chi=\epsilon$ for $a=$ ord. For $a=$ norm, $\chi=\phi$ if $\langle\phi\rangle_{P}$ is nonvanishing, and $\chi=\epsilon$ otherwise.

Using the boundary operator expansion ${ }^{6-9}$ near the planar surface,

$$
\begin{aligned}
\psi\left(r_{1 \perp}, \mathbf{r}_{1 \|}\right)= & A_{\psi}^{(a)}\left(2 r_{1 \perp}\right)^{-x} \psi\left[1-x_{\psi} C^{(a)} r_{1 \perp}^{d} T_{n n}\left(r_{1 \perp} \rightarrow 0, \mathbf{r}_{1 \|}\right)\right. \\
& +\ldots]
\end{aligned}
$$

for the half-space functions in Eq. (2.4) yields

$$
X_{\psi, \chi}^{(a)}(y) \rightarrow \beta_{\psi}^{(a)} \gamma_{\chi}^{(a)} y^{d}, \quad y \rightarrow 0
$$

with

$$
\beta_{\psi}^{(a)}=\left(A_{\psi}^{(a)} / \sqrt{B_{\psi}}\right) x_{\psi} C^{(a)} / 2^{d}, \quad \gamma_{\chi}^{(a)}=\left(A_{\chi}^{(a)} / \sqrt{B_{\chi}}\right) x_{\chi} / \Omega_{d} .
$$

Equations (2.1), (2.2), and (2.6) then lead to the profile correction

$$
\delta_{P}\langle\psi\rangle_{S} / \sqrt{B_{\psi}} \rightarrow(2(r-R))^{d-x_{\psi}} \beta_{\psi}^{(a)} \tau_{S, P}, \quad l \ll r-R \ll R,
$$

where $^{23}$

$\tau_{S, P}=\sum_{\chi=\phi, \epsilon} \gamma_{\chi}^{(a)} R^{x} \chi^{-d}\left[1+\frac{d}{x_{\chi}} \mathbf{r} \frac{\partial}{\partial \mathbf{r}_{c}}\right] \delta_{P}\left\langle\chi\left(\mathbf{r}_{c}\right)\right\rangle_{\mathrm{bulk}} / \sqrt{B_{\chi}}, \quad r=R$

is independent of $\psi$. Here $l$ denotes microscopic lengths and $\Omega_{d}=2 \pi^{d / 2} / \Gamma(d / 2)$ is the surface area of a sphere of radius 1 . The amplitude $C^{(a)}$ depends on $a$ in general, but for $a= \pm$ is the same for $\psi=\phi$ or $\epsilon$. Numerical estimates are given in the two papers of Ref. 8. In $d=2, C^{(a)}=4 \pi / c$ is independent ${ }^{5,6}$ of $a$ and is completely determined by the conformal charge $c$, which is a bulk quantity and equals $1 / 2$ for the Ising model. As noted in the paragraph above Sec. II A, for the energy density near a normal sphere and a symmetry breaking perturbation $\langle\phi\rangle_{P} \neq 0$, it is the nondiagonal contribution $\chi=\phi$ that dominates the correction $\delta_{P}\langle\epsilon\rangle_{S}$.

The relation to the force is provided by the integral

$$
\int \mathrm{d} \mathbf{S} \tau_{S, P} \rightarrow \frac{\mathbf{f}}{k_{B} T}
$$

over the surface, which tends to $\mathbf{f}$ in Eq. (3.2). Here the vectorial surface element $\mathrm{d} \mathbf{S}$ points away from the sphere center, and we have used that $R \int \mathrm{d} S_{k}\left(r_{l} / r\right)$ equals $R^{d} \Omega_{d} \delta_{l k} / d$.

For $P$ the planar boundary of the half space $-\infty<r_{\perp}$ $<\mathcal{D}$ with the sphere center at $r_{\perp}=0$, as shown in Fig. 1, and the bulk system right at the critical point,

$$
\left(1, \frac{\mathbf{r}}{x_{\chi}} \frac{\partial}{\partial \mathbf{r}_{c}}\right) \delta_{P}\left\langle\chi\left(\mathbf{r}_{c}\right)\right\rangle_{\text {bulk }}=\left(1, \frac{r_{\perp}}{\mathcal{D}}\right) \frac{A_{\chi}^{(b)}}{(2 \mathcal{D})^{x} \chi},
$$

and Eq. (3.7) yields

$\tau_{S, P}=\sum_{\chi=\phi, \epsilon} \gamma_{\chi}^{(a)}\left(A_{\chi}^{(b)} / \sqrt{B_{\chi}}\right)(2 \mathcal{D})^{-x} \chi R^{x} \chi^{-d}\left[1+d \frac{r_{\perp}}{\mathcal{D}}\right], \quad r=R$.

Here $b$ is the surface class of the wall $P$. This should be compared with the parallel plate geometry, ${ }^{5-8}$ where walls $W$ and $P$ with surface classes $a$ and $b$ are located at $r_{\perp}=0$ and $r_{\perp}=\mathcal{D}$, and where the profile correction for $0<r_{\perp} \ll \mathcal{D}$ is given by

$$
\delta_{P}\langle\psi\rangle_{W} / \sqrt{B_{\psi}} \rightarrow\left(2 r_{\perp}\right)^{d-x} \psi \beta_{\psi}^{(a)} \tau_{W, P}
$$

where $^{23}$

$$
\tau_{W, P}=-(d-1) \Delta_{a, b} \mathcal{D}^{-d}
$$

is the Casimir force per unit area and $k_{B} T$ on the wall $W$ due to the presence of $P$. 
The behavior of $\delta_{P}\langle\psi\rangle_{S}$ and $\delta_{P}\langle\psi\rangle_{W}$ is qualitatively similar. In particular, for a given pair $(a, b)$ of surface classes, the signs

$$
\operatorname{sgn} \tau_{S, P}=\operatorname{sgn} \tau_{W, P}
$$

are the same. For equal classes $a=b=+,-$ or "ord," the signs are +1 , and for the unequal classes $(a, b)=(+,-),(-,+)$, ( \pm , ord), or (ord, \pm ), the signs are -1 . Since the anisotropic term $\propto r_{\perp}$ in Eq. (3.10) strengthens (weakens) the isotropic effect on the side of the sphere which is closer to (farther from) the perturbing planar wall $P$, for a given pair $(a, b)$ the forces on $S$ in Eq. (3.8) and on $W$ in Eq. (3.12) also have the same sign, both pointing either toward or away from $P$, i.e., in both cases an attractive or repulsive interaction for equal or unequal surface classes, respectively.

However, other important features are different. In contrast to the case of parallel plates, in which only the amplitude in the power law of $\tau_{W, P}$ depends on the surface classes $(a, b)$ in the sphere-wall case, the exponents in the leading behavior $\propto \mathcal{D}^{-x} x R^{x^{x}}-d$ of $\tau_{S, P}$ also depend on $(a, b)$. For example, $x_{\chi}=x_{\phi}$ for $a=+, b= \pm$, while $x_{\chi}=x_{\epsilon}$ for $a=+, b=$ ord .

\section{B. Corrections far from the surface and the small sphere expansion}

For $r \gg R$, i.e., $y \gg 1$, the behavior of $X_{\psi, \chi}^{(a)}$ in Eq. (2.2) is determined by the half-space cumulants in Eq. (2.4) near the bulk limit, where

$$
\begin{aligned}
& X_{\psi, \psi}^{(a)}(y)-y^{x_{\psi}} \rightarrow y^{x_{\psi^{-}}\left(x_{\epsilon} / 2\right)} C_{\psi \psi \epsilon^{\prime}} A_{\epsilon}^{(a)} /\left(B_{\psi} B_{\epsilon}\right)-\left(A_{\psi}^{(a)}\right)^{2} / B_{\psi}, \\
& X_{\phi, \epsilon}^{( \pm)}(y) \rightarrow y^{x_{\epsilon} / 2} C_{\phi \phi \epsilon} A_{\phi}^{( \pm)} /\left(B_{\phi}^{3 / 2} B_{\epsilon}^{1 / 2}\right) .
\end{aligned}
$$

This follows from expansions for "short" distances $\left|\mathbf{r}_{1}-\mathbf{r}_{2}\right|$ of the operator products $\psi\left(\mathbf{r}_{1}\right) \chi\left(\mathbf{r}_{2}\right)$, compare Appendix B. Here $C_{\psi_{1} \psi_{2} \psi_{3}}$ is the amplitude in the three-point function in the bulk at the critical point, $\left\langle\psi_{1}\left(\mathbf{r}_{1}\right) \psi_{2}\left(\mathbf{r}_{2}\right) \psi_{3}\left(\mathbf{r}_{3}\right)\right\rangle_{\text {bulk }}$, which equals $C_{\psi_{1} \psi_{2} \psi_{3}} /\left(r_{12}^{x_{1}+x_{2}-x_{3}} r_{23}^{x_{2}+x_{3}-x_{1}} r_{31}^{\left.x_{3}+x_{1}-x_{2}\right)}\right.$. Due to $x_{\epsilon} \geq 2 x_{\phi}$, the $y$-independent term in the first Eq. (3.14) is important for $\psi=\phi$, while it can be neglected for $\psi=\epsilon$.

In the limit $R \rightarrow 0$, the right-hand sides of the two Eqs. (3.14) do not contribute to $\mathcal{F}$ in Eqs. (2.1)-(2.3), and one finds ${ }^{24}$

$$
\lim _{R \rightarrow 0} \mathcal{F}^{(\psi, a)}\left(R, \mathbf{r} ; \mathbf{r}_{c}\right) \rightarrow\left[1+\mathbf{r} \frac{\partial}{\partial \mathbf{r}_{c}}\right] \psi\left(\mathbf{r}_{c}\right) / \sqrt{B_{\psi}},
$$

i.e., the Taylor expansion of $\psi\left(\mathbf{r}_{c}+\mathbf{r}\right) / \sqrt{B_{\psi}}$, as expected.

Now we consider the correction (2.6) of the density profile and show that besides $y^{x} \psi$ the other terms in Eq. (3.14) are also consistent with the form

$$
\left\langle\psi\left(\mathbf{r}_{c}+\mathbf{r}\right)\right\rangle_{S, P}=\left\langle\psi\left(\mathbf{r}_{c}+\mathbf{r}\right)(1+\sigma)\right\rangle_{P} /\left(1+\langle\sigma\rangle_{P}\right)
$$

of the density profile that follows on using the small sphere expansion (SSE) in Eq. (2.8) for $S$. Here we consider the perturbation $P$ from a single distant particle or wall with surface class $b$, and we confine our attention to the leading isotropic and anisotropic contributions as the smallest distance $\mathcal{D}$ between $\mathbf{r}_{c}$ and $P$ becomes large. In the fusion equation (2.6) these contributions are given by $\chi=\phi$ for

$$
(a, \psi ; b)=(\operatorname{ord}, \phi ;+) ;(+, \phi ; \pm),(+, \epsilon ; \pm)
$$

and by $\chi=\epsilon$ for

$$
\begin{aligned}
(a, \psi ; b)= & (\text { ord }, \epsilon ; \text { ord }),(\text { ord }, \epsilon ;+) ;(+, \phi ; \text { ord }), \\
& (+, \epsilon ; \text { ord }) .
\end{aligned}
$$

The terms $\propto C_{\psi_{1} \psi_{2} \psi_{3}}$ in the fusion-scheme (2.6) that arise from Eq. (3.14) are reproduced in the SSE scheme of Eq. (3.16) by inserting the operator product expansions of Eq. (B1) in the average $\langle\psi \sigma\rangle$ of the first order contribution in $\sigma$,

$$
\begin{aligned}
\left.\delta_{P}\left\langle\psi\left(\mathbf{r}_{c}+\mathbf{r}\right)\right\rangle_{S}\right|_{\mathcal{O}(\sigma)}= & \delta_{P}\left[\left\langle\psi\left(\mathbf{r}_{c}+\mathbf{r}\right) \sigma\right\rangle\right. \\
& \left.-\left\langle\psi\left(\mathbf{r}_{c}+\mathbf{r}\right)\right\rangle\langle\sigma\rangle\right]_{\text {bulk }} .
\end{aligned}
$$

Here the leading terms are given by $\sigma=\sigma_{\phi}$ for

$$
(a, \psi ; b)=(+, \phi ; \text { ord }),(+, \epsilon ; \pm)
$$

and by $\sigma=\sigma_{\epsilon}$ for

$$
\begin{aligned}
(a, \psi ; b)= & (\text { ord }, \phi ;+),(\text { ord }, \epsilon ; \text { ord }),(\text { ord }, \epsilon ;+) ; \\
& (+, \phi ; \pm),(+, \epsilon ; \text { ord }) .
\end{aligned}
$$

A possible operator-free term $\propto r^{-2 x_{\psi}}$ drops out of $\delta_{P}\langle\psi \sigma\rangle$, and $\delta_{P}[\langle\psi\rangle\langle\sigma\rangle]$ in Eq. (3.19) can be neglected for large $\mathcal{D}$ and $\xi$.

Finally, consider the $y$-independent term $-\left(A_{\psi}^{(a)}\right)^{2} / B_{\psi}$ in Eq. (3.14). It is of the same order as or even dominates the term $\propto C_{\psi \psi \epsilon}$ in the cases $(a, \psi ; b)$ equal to $(+, \phi ; \pm)$, since $x_{\phi}-\left(x_{\epsilon} / 2\right) \leq 0$, and equal to (ord, $\epsilon$; ord) in the $d=2$ Ising model, ${ }^{26}$ since $C_{\epsilon \epsilon \epsilon}$ vanishes. Its contribution to the fusion in Eq. (2.6) is reproduced in the SSE scheme by the second order term in $\sigma$ of Eq. (3.16),

$$
\begin{aligned}
\left.\delta_{P}\left\langle\psi\left(\mathbf{r}_{c}+\mathbf{r}\right)\right\rangle_{S}\right|_{\mathcal{O}\left(\sigma^{2}\right)}= & -\delta_{P}\left[\left\langle\psi\left(\mathbf{r}_{c}+\mathbf{r}\right) \sigma\right\rangle\langle\sigma\rangle\right. \\
& \left.-\left\langle\psi\left(\mathbf{r}_{c}+\mathbf{r}\right)\right\rangle\langle\sigma\rangle^{2}\right]_{\mathrm{bulk}},
\end{aligned}
$$

which due to $\left\langle\psi\left(\mathbf{r}_{c}+\mathbf{r}\right) \psi\left(\mathbf{r}_{c}\right)\right\rangle \rightarrow B_{\psi} / r^{2 x} \psi$ yields

$$
-\frac{1}{r^{2 x_{\psi}}} A_{\psi}^{(a)} R^{x_{\psi}} \delta_{P}\left\langle\sigma_{\psi}\right\rangle_{\mathrm{bulk}}=-\frac{\left(A_{\psi}^{(a)}\right)^{2}}{B_{\psi}}\left(\frac{R}{r}\right)^{2 x_{\psi}} \delta_{P}\left\langle\psi\left(\mathbf{r}_{c}\right)\right\rangle_{\mathrm{bulk}}
$$

in leading order.

\section{DERIVING THE FUSION BY A CONFORMAL TRANSFORMATION}

If the system is right at the critical point, one may exploit conformal invariance to check the fusion relation (2.6) or (2.7). The special conformal transformation

$$
\frac{\mathbf{r}^{\prime}}{r^{\prime 2}}=\frac{\mathbf{r}+\mathbf{R}}{|\mathbf{r}+\mathbf{R}|^{2}}-\frac{\mathbf{R}}{2 R^{2}}
$$

with an arbitrary vector $\mathbf{R}$ of length $R$ maps the sphere $S$ of radius $R$ and center at $\mathbf{r}=\mathbf{0}$ onto the plane $r_{\perp}^{\prime} \equiv-\mathbf{r}^{\prime} \mathbf{R} / R=0$ and the exterior of $S$ onto the half space $r_{\perp}^{\prime}>0$. As the distance of the perturbation $P$ from $S$ becomes larger and larger, the image $P^{\prime}$ of $P$ shrinks to the point $\mathbf{r}^{\prime}=-2 \mathbf{R}$, which is the image of $\mathbf{r}=\infty$, and one can apply the small particle expansion to $P^{\prime}$. On transforming back to $\mathbf{r}$-space, this leads to the form (2.6) of the density correction, as we shall see. 
We consider in detail the case in which $P$ is a spherical particle with radius $R_{P}$ and surface class $b$. Placing the center of $P$ at

$$
\mathbf{r}_{c P}=\mathbf{r}_{c}+\mathbf{r}_{P}, \quad \mathbf{r}_{P}=-r_{P} \mathbf{R} / R
$$

yields

$$
\left(1, \frac{\mathbf{r}}{x_{\chi}} \frac{\partial}{\partial \mathbf{r}_{c}}\right) \delta_{P}\left\langle\chi\left(\mathbf{r}_{c}\right)\right\rangle_{\text {bulk }}=\left(1,2 \frac{\mathbf{r r}_{P}}{r_{P}^{2}-R_{P}^{2}}\right) A_{\chi}^{(b)}\left(\frac{R_{P}}{r_{P}^{2}-R_{P}^{2}}\right)^{x_{\chi}}
$$

for the two expressions on the right-hand side of Eq. (2.6). Here we use the fact that at the critical point $\langle\chi\rangle_{\text {bulk }}$ vanishes, and the density profile $\langle\chi\rangle_{P}$ around a spherical particle $P$ has the form derived in Ref. 20. Note that the differentiation $\partial / \partial \mathbf{r}_{c}$ is performed at fixed $\mathbf{r}_{c P}$.

Now we set $\mathbf{r}_{c}=0$ for simplicity and calculate the lefthand side of Eq. (2.6) by means of the conformal transformation (4.1), which on using Appendix (C3) yields

$$
\delta_{P}\langle\psi(\mathbf{r})\rangle_{S}=\left[b\left(\mathbf{r}^{\prime}\right)\right]^{x} \psi\left\{\left\langle\psi\left(\mathbf{r}^{\prime}\right)\right\rangle_{S^{\prime}, P^{\prime}}-\left\langle\psi\left(\mathbf{r}^{\prime}\right)\right\rangle_{S^{\prime}}\right\} .
$$

Here $b\left(\mathbf{r}^{\prime}\right)$ is introduced in Eq. (C2) and $\left(S^{\prime}, P^{\prime}\right)$ and $S^{\prime}$ denote the half space $r_{\perp}^{\prime}>0$ in the presence and absence of $P^{\prime}$,

respectively. The image $P^{\prime}$ of the sphere $P$ with center at $\mathbf{r}=\mathbf{r}_{P}$ is a spherical particle with radius

$$
R_{P^{\prime}}=R(\lambda-\mu)
$$

center at

$$
\mathbf{r}^{\prime}=\mathbf{r}_{P^{\prime}}^{\prime}=-\mathbf{R}(\lambda+\mu)
$$

and surface class $b$. Here

$$
\lambda=\frac{r_{P}+R_{P}-R}{r_{P}+R_{P}+R}, \quad \mu=\frac{r_{P}-R_{P}-R}{r_{P}-R_{P}+R}
$$

Note that $\mathbf{r}_{P^{\prime}}^{\prime}$ is not the image of $\mathbf{r}_{P}$, but instead the image of the point

$$
\mathbf{r}_{P^{\prime}}=-\mathbf{R} \frac{2+\lambda+\mu}{2-(\lambda+\mu)}
$$

in r-space, see Eq. (C1).

In the special case that $P$ is a planar wall with the shortest distance $\mathcal{D}=r_{P}-R_{P}$ from the center of $S$, both $r_{P}$ and $R_{P}$ are infinitely large, and Eq. (4.3) reduces to Eq. (3.9), where $r_{\perp}$ is equal to $\mathbf{r r}_{P} / r_{P}$ and the planar wall is located at $r_{\perp}=\mathcal{D}$. In this case $\lambda=1$ and $\mu=(\mathcal{D}-R) /(\mathcal{D}+R)$, so that $\mathbf{r}_{P^{\prime}}^{\prime}=-2 \mathbf{R} \mathcal{D} /(\mathcal{D}+R)$ and $R_{P^{\prime}}=2 R^{2} /(\mathcal{D}+R)$. Note that $\mathbf{r}_{P^{\prime}}=-\mathbf{R}((2 \mathcal{D} / R)+1)$ is finite while $\mathbf{r}_{P}=\infty$.
Using in Eq. (4.4) the small sphere expansion for $P^{\prime}$ that follows from Eq. (2.8) on replacing $a, R$, and $\mathbf{r}_{c}$ by $b, R_{P^{\prime}}$, and $\mathbf{r}_{P^{\prime}}^{\prime}$ yields

$$
\begin{aligned}
\delta_{P}\langle\psi(\mathbf{r})\rangle_{S} / \sqrt{B_{\psi}}= & {\left[b\left(\mathbf{r}^{\prime}\right)\right]^{x} \psi\left(2 r_{\perp}^{\prime}\right)^{-x} \psi } \\
& \times \sum_{\chi=\phi, \epsilon} \frac{A_{\chi}^{(b)}}{\sqrt{B_{\chi}}}\left(R_{P^{\prime}}\right)^{x} \chi\left(2 r_{P^{\prime} \perp}^{\prime}\right)^{-x} \chi \\
& \times X_{\psi, \chi}^{(a)}\left(\frac{4 r_{\perp}^{\prime} r_{P^{\prime} \perp}^{\prime}}{\left|\mathbf{r}^{\prime}-\mathbf{r}_{P^{\prime}}^{\prime}\right|^{2}}\right) \\
= & (R y)^{-x} \psi \sum_{\chi=\phi, \epsilon} \frac{A_{\chi}^{(b)}}{\sqrt{B_{\chi}}}\left(\frac{\lambda-\mu}{2(\lambda+\mu)}\right)^{x_{\chi}} \\
& \times X_{\psi, \chi}^{(a)}\left(y \frac{r_{P^{\prime}}^{2}-R^{2}}{\left|\mathbf{r}_{P^{\prime}}-\mathbf{r}\right|^{2}}\right),
\end{aligned}
$$

where $y$ is from Eq. (2.3). In the first step of Eq. (4.9), the form (2.4) of the two-point cumulant in the half space has been taken into account, and in the second step, we have used Eqs. (4.5), (4.6), (C4), and (C5). In order to obtain the leading isotropic and anisotropic $\mathbf{r}$-dependence for a distant sphere $P$ where $r_{P}-R_{P}$ and $r_{P}+R_{P}$ are much larger than $R$, we expand the two expressions

$$
\frac{\lambda-\mu}{2(\lambda+\mu)} \rightarrow \frac{R R_{P}}{r_{P}^{2}-R_{P}^{2}}
$$

and

$$
\frac{r_{P^{\prime}}^{2}-R^{2}}{\left|\mathbf{r}_{P^{\prime}}-\mathbf{r}\right|^{2}} \rightarrow 1+2 \frac{\mathbf{r r}_{P}}{r_{P}^{2}-R_{P}^{2}}
$$

that appear in Eq. (4.9). Finally, expanding $X^{(a)}$ to first order in the small quantity $\mathbf{r r}_{P} /\left(r_{P}^{2}-R_{P}^{2}\right)$ and taking the identities (2.2) and (4.3) into account, one confirms that $\delta_{P}\langle\psi\rangle_{S}$ in Eq.

(4.9) does indeed obey the fusion relation (2.6).

\section{ANALYTIC EXPRESSIONS FOR PROFILE CORRECTIONS}

In some cases there exist closed form expressions for the half space functions $X_{\psi, \chi}^{(a)}(y)$ in Eq. (2.4), implying exact explicit expressions for the profile corrections $\delta_{P}\langle\psi\rangle_{S}$. Here we consider the geometry addressed above Eq. (3.9) and shown in Fig. 1 of a planar wall $P$ at a distance $\mathcal{D}$ from the center of $S$ and calculate the profiles in the asymptotic regime $R, r$ $\ll \mathcal{D}$ with the bulk right at the critical point. The results

$$
\begin{aligned}
\delta_{P}\langle\psi\rangle_{S} / \sqrt{B_{\psi}} \rightarrow & {\left[\mathcal{A}_{\kappa}^{(\psi, a)}+\tilde{\mathcal{A}}_{\kappa}^{(\psi, a)} x_{\kappa} r_{\perp} / \mathcal{D}\right] \vartheta_{\kappa}^{(b)} } \\
\equiv & R^{-x_{\psi}}\left[\alpha_{\kappa}^{(\psi, a ; b)}(r / R) \varrho^{x_{\kappa}}\right. \\
& \left.+\left(r_{\perp} / R\right) \widetilde{\alpha}_{\kappa}^{(\psi, a ; b)}(r / R) \varrho^{x_{\kappa}+1}\right],
\end{aligned}
$$

where

$$
\vartheta_{\kappa}^{(b)}=\left(A_{\kappa}^{(b)} / \sqrt{B_{\kappa}}\right)(2 \mathcal{D})^{-x_{\kappa}}, \quad \varrho=R /(2 \mathcal{D}),
$$

follow from Eqs. (2.6) and (3.9). Here $\chi=\kappa$ is the field in the sum in Eq. (2.6), which yields the leading contribution for $R \ll \mathcal{D}$. 


\section{A. Ordinary sphere surface $(a=$ ord $)$}

Now we consider spheres with a symmetry preserving surface $a=$ ord, where only $\chi=\psi$ contributes in Eq. (2.6).

(1) We start with the energy density $\psi=\epsilon$ in the Gaussian model in $d>2$ perturbed by a wall with surface class $b=$ ord. For this case Eqs. (2.19) and (2.15) yield

$$
\begin{aligned}
\delta_{P}\langle\epsilon\rangle_{S} / \sqrt{B_{\epsilon}} \rightarrow & \left(1-q^{x_{\epsilon}}\right)\left[1-q^{x_{\epsilon}}+\left(1-q^{x_{\epsilon}+2}\right) x_{\epsilon} r_{\perp} / \mathcal{D}\right] \\
& \times \vartheta_{\epsilon}^{\text {(ord) }},
\end{aligned}
$$

where $^{22}$

$\vartheta_{\epsilon}^{\text {(ord) }}=2^{-1 / 2}(2 \mathcal{D})^{-x_{\epsilon}}, \quad x_{\epsilon}=d-2$

and

$q=R / r$.

(2) In the $d=2$ Ising model, ${ }^{26}$ where the nanoparticle is a circular disk, the corresponding profile correction follows from the results in Refs. 5 and 14 and reads

$\delta_{P}\langle\epsilon\rangle_{S} / \sqrt{B_{\epsilon}} \rightarrow\left(1-q^{2}\right)\left[1+\left(1+q^{2}\right) r_{\perp} / \mathcal{D}\right] \vartheta_{\epsilon}^{\text {(ord) }}$,

where $^{26}$

$\vartheta_{\epsilon}^{\text {(ord) }}=(2 \mathcal{D})^{-1}$.

(3) For the order parameter in the $d=2$ Ising model perturbed by a wall with $b=+$,

$$
\begin{aligned}
\delta_{P}\langle\phi\rangle_{S} / \sqrt{B_{\phi}} \rightarrow & \left(1-q^{2}\right)^{-1 / 8}(1-q)^{1 / 2} \\
& \times\left[1+(1+q)^{2} r_{\perp} /(8 \mathcal{D})\right] \vartheta_{\phi}^{(+)},
\end{aligned}
$$

where ${ }^{26}$

$$
\vartheta_{\phi}^{(+)}=2^{1 / 4}(2 \mathcal{D})^{-1 / 8} \text {. }
$$

\section{B. Normal sphere surface $(a=+)$}

In the Ising model we consider spheres with surface spins fixed in the positive spin direction and walls with $b=+$ or $b=-$, corresponding to spins fixed in the positive or negative direction. For both $\delta_{P}\langle\phi\rangle_{S}$ and $\delta_{P}\langle\epsilon\rangle_{S}, \kappa$ equals $\phi$.

(4), (5) For $d \nearrow 4$, Landau mean field theory can be applied and the corrections of the order parameter and energy density profiles are given by ${ }^{27}$

$$
\begin{aligned}
& \delta_{P}\left\{\langle\phi\rangle_{S} / \sqrt{B_{\phi}},\langle\epsilon\rangle_{S} / \sqrt{B_{\epsilon}}\right\} \\
& \rightarrow\left\{1,-\frac{6 \sqrt{2}}{\sqrt{\varepsilon}} \frac{R}{r^{2}} \frac{1}{1-q^{2}}\right\} \\
& \times\left[1-q^{2}+12 \frac{q^{2}}{1-q^{2}}\left(1+\frac{1+q^{2}}{1-q^{2}} \ln q\right)\right. \\
&+\left(1-q^{4}-6 q^{2} \frac{1+q^{2}}{1-q^{2}}\right. \\
&\left.\left.-24 \frac{q^{4}}{\left(1-q^{2}\right)^{2}} \ln q\right) \frac{r_{\perp}}{\mathcal{D}}\right] \vartheta_{\phi}^{(b)},
\end{aligned}
$$

where

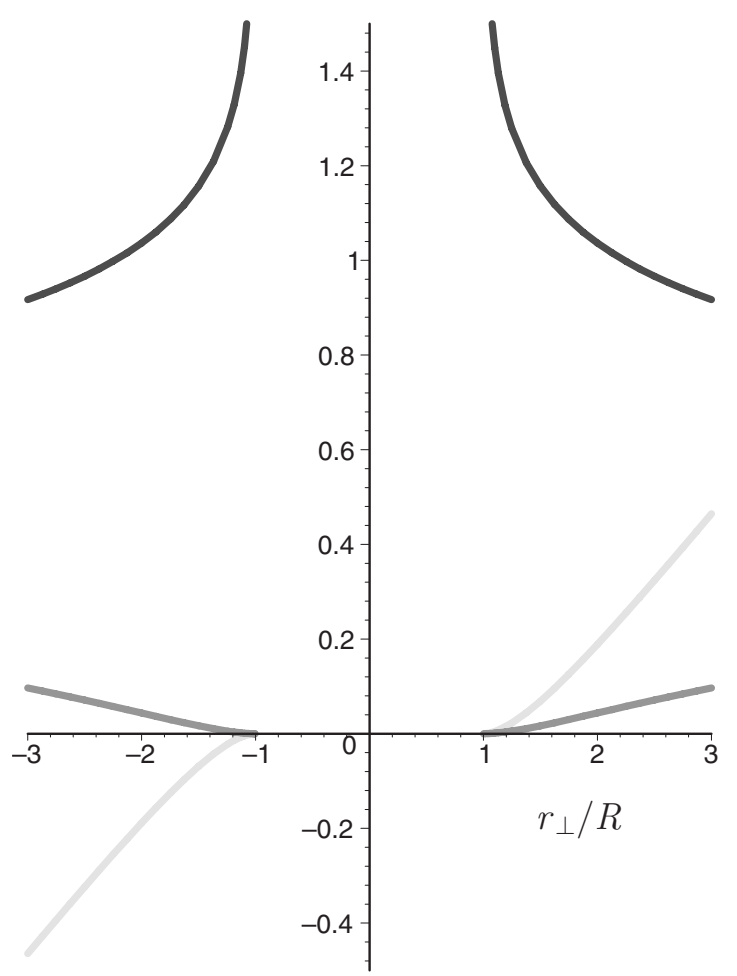

FIG. 2. Functions entering the dimensionless order parameter profile $R^{x} \phi\left[\langle\phi\rangle_{S}+\delta_{P}\langle\phi\rangle_{S}\right] / \sqrt{B_{\phi}}$ for a circular disk $S$ and perturbing wall $P$ with $a=b=+$ in the $d=2$ Ising model along the line $r_{\|}=0$. Shown are the unperturbed contribution $R^{x} \phi\langle\phi\rangle_{S} / \sqrt{B_{\phi}}$ (upper curve) and the functions $\alpha_{+}^{(\phi,+;)}\left(\left|r_{\perp}\right| / R\right)$ and $\left(r_{\perp} / R\right) \widetilde{\alpha}_{+}^{(\phi,+;+)}\left(\left|r_{\perp}\right| / R\right)$ in Eq. (5.1) (lower curves even and odd in $\left.r_{\perp} / R\right)$ which determine the leading isotropic and anisotropic perturbations from the distant wall and follow from Eq. (5.12).

$$
\vartheta_{\phi}^{(+)}=3 \varepsilon^{-1 / 2} \mathcal{D}^{-1}, \quad \vartheta_{\phi}^{(-)}=-\vartheta_{\phi}^{(+)},
$$

and $\varepsilon=4-d$.

(6) For the $d=2$ Ising model, the necessary half-space functions $X$ can be taken from Refs. 5 and 14, and for the order parameter this yields

$$
\begin{aligned}
\delta_{P}\langle\phi\rangle_{S} / \sqrt{B_{\phi}} \rightarrow & \left(1-q^{2}\right)^{-1 / 8}\left[(1+q)^{1 / 2}-2^{1 / 2} q^{1 / 4}\right. \\
& \left.+(1-q)^{2}(1+q)^{1 / 2} r_{\perp} /(8 \mathcal{D})\right] \vartheta_{\phi}^{(b)},
\end{aligned}
$$

where $\vartheta_{\phi}^{+}$is given in Eq. (5.9) and $\vartheta_{\phi}^{(-)}=-\vartheta_{\phi}^{(+)}$. Figures 2 and 3 show the behavior of the dimensionless profile $R^{x} \phi\left[\langle\phi\rangle_{S}+\delta_{P}\langle\phi\rangle_{S}\right] / \sqrt{B_{\phi}}$ along the line $r_{\|}=0$ in Fig. 1 that is perpendicular to the wall and passes through the center of the circular disk $S$.

(7) For the energy density in $d=2$ one finds

$$
\begin{aligned}
\delta_{P}\langle\epsilon\rangle_{S} / \sqrt{B_{\epsilon}} \rightarrow & -2^{-3 / 4}\left(R^{1 / 8} / r\right)(1-q)\left[(1+q)^{-1}\right. \\
& \left.+(1+q) r_{\perp} /(2 \mathcal{D})\right] \vartheta_{\phi}^{(b)} .
\end{aligned}
$$

In all these examples $\widetilde{\mathcal{A}}_{\kappa}^{(\psi, a)} x_{\kappa} / \mathcal{A}_{\kappa}^{(\psi, a)}=\mathrm{d} \ln X_{\psi, \kappa}^{(a)} / \mathrm{d} \ln y$ is positive and, as expected intuitively, the effect of the isotropic correction is enhanced (reduced) by the anisotropic correction for $r_{\perp}>0\left(r_{\perp}<0\right)$, i.e., for points closer to (farther from) the perturbing wall, compare Figs. 2 and 3. For $q \nearrow 1$ and $q \searrow 0$ the closed form expressions reproduce, of course, 


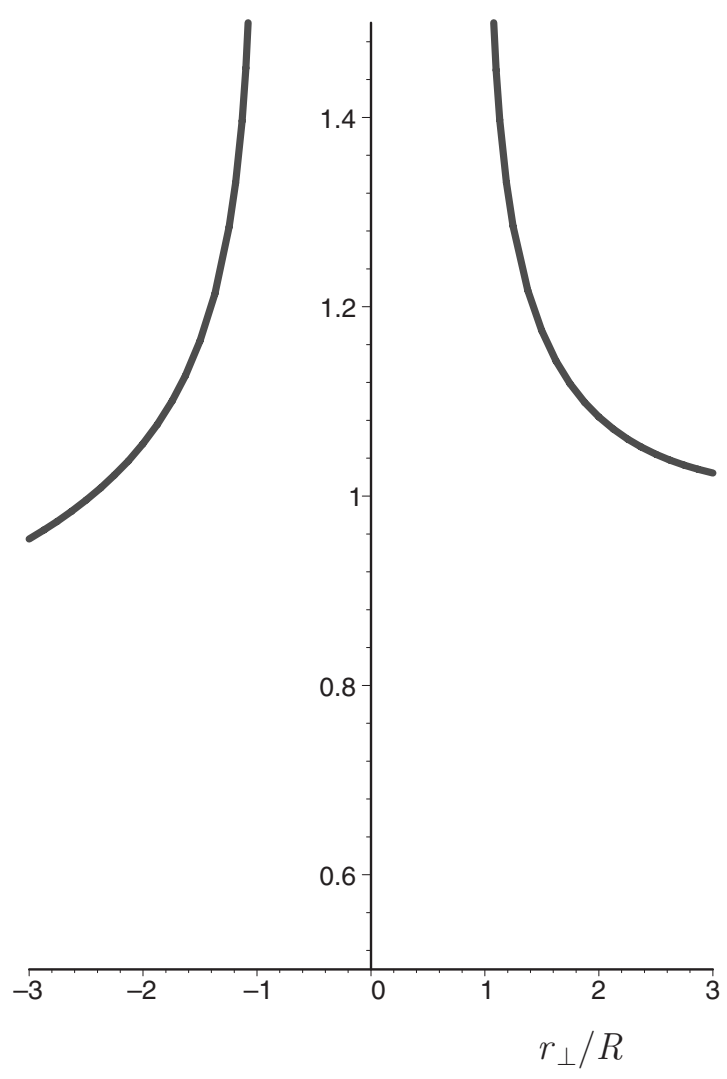

FIG. 3. Dimensionless order parameter profile along the line $r_{\|}=0$ for a disk and perturbing wall with $a=b=+$ in the $d=2$ Ising model as given by the sum of the three functions in Fig. 2 after multiplying with $1, \varrho^{x} \phi$, and $\varrho^{x} \phi^{+1}$, respectively, compare Eq. (5.1). Shown is the case $\varrho \equiv R /(2 \mathcal{D})=1 / 10$. As expected, the profile is larger (smaller) for points with positive (negative) $r_{\perp}$, which are closer to (farther from) the perturbing wall at $r_{\perp}=\mathcal{D}$.

the limiting behavior close to and far from the surface of $S$, which have been discussed in Secs. III A and III B, respectively.

\section{DENSITIES, PRESSURE, AND FORCE OF NONADSORBING POLYMERS}

Now we consider the nanosphere $S$ embedded in a dilute monodisperse solution of long, flexible, nonadsorbing polymer chains and study the density profiles $\mathcal{M}_{S, P}$ and $\mathcal{E}_{S, P}$ of chain segments and chain end points in the presence of a distant perturbation $P$ such as a planar wall or another particle. We normalize $\mathcal{M}$ and $\mathcal{E}$ to be 1 in the bulk, far from $S$ and $P$. For nonadsorbing polymers the surfaces of $S$ and $P$ are repulsive and belong to the "ordinary" surface class. ${ }^{13}$ For $R, r \ll \mathcal{D}, \mathcal{R}_{g}$ with $\mathcal{R}_{g}$ the radius of gyration of one chain, the densities are given by

$$
\mathcal{K}_{S, P}\left(\mathbf{r}_{c}+\mathbf{r}\right) \rightarrow\left\{\mathcal{A}_{\psi}^{(\psi, \text { ord })}+\tilde{\mathcal{A}}_{\psi}^{(\psi, \text { ord })} \mathbf{r} \frac{\partial}{\partial \mathbf{r}_{c}}\right\} \mathcal{K}_{P}\left(\mathbf{r}_{c}\right),
$$

where

$$
(\mathcal{K}, \psi)=(\mathcal{M}, \epsilon) \text { or } \quad(\mathcal{E}, \phi),
$$

and $\mathcal{M}_{P}$ and $\mathcal{E}_{P}$ are the densities in the absence of $S$. This is shown in Appendix D.

For "ideal" chains without excluded volume interactions between chain segments, the amplitude functions $\mathcal{A}$ and $\tilde{\mathcal{A}}$ are those of the Gaussian model, given in Eqs. (2.15) and (2.19). For "real" chains with excluded volume interactions, the amplitudes follow via Eqs. (2.2)-(2.4) from the two-point cumulants of the $N \rightarrow 0$ vector model ${ }^{13,28}$ in the half space. In $d=2$ exact results can be obtained for $\mathcal{A}_{\epsilon}^{(\epsilon, \text { ord })}$ and $\widetilde{\mathcal{A}}_{\epsilon}^{(\epsilon, \text { ord })}$ via Eqs. (2.2) and (2.3) from ${ }^{29}$

$$
X_{\epsilon, \epsilon}^{\text {(ord) }}(y)=\frac{8 \pi}{45 \sqrt{3}} \frac{y^{2}}{y+1}{ }_{3} F_{2}\left(\frac{1}{3}, \frac{2}{3}, \frac{7}{3} ; \frac{11}{6}, 2 ; \frac{-y^{2}}{4(1+y)}\right),
$$

where ${ }_{3} F_{2}$ is a hypergeometric function. The form (6.3) applies for $y$ and thus $r / R$ not too large and requires analytic continuation for larger $y$ and $r / R$.

For $\mathcal{D} \rightarrow \infty$, Eq. (6.1) reproduces the results $\mathcal{K}=\mathcal{K}_{S}=\mathcal{A}_{\psi}^{(\psi, \text { ord })}$ for the densities around a nanosphere in the bulk solution, which were obtained in Ref. 10. Due to Eq. (2.7), $\mathcal{A}_{\psi}^{(\psi, \text { ord })}$ equals the limit of the ratio of $\left\langle\psi\left(\mathbf{r}_{1}\right) \psi\left(\mathbf{r}_{c}+\mathbf{r}\right)\right\rangle_{S}$ at the critical point and $B_{\psi} r_{1}^{-2 x_{\psi}}$ as $r_{1} \rightarrow \infty$. This representation for $\mathcal{K}_{S}$ also applies to nonspherical ${ }^{10,30}$ nanoparticles $S$, see in particular Eqs. (3.2) and (3.1) in Ref. 30

Note that the product approximation ${ }^{31} \mathcal{K}_{S}\left(\mathbf{r}_{c}+\mathbf{r}\right) \mathcal{K}_{P}$ $\left(\mathbf{r}_{c}+\mathbf{r}\right)$ for $\mathcal{K}_{S, P}\left(\mathbf{r}_{c}+\mathbf{r}\right)$ leads in our case $R, r \ll \mathcal{D}, \mathcal{R}_{g}$ to $\mathcal{A}_{\psi}^{(\psi \text {,ord })}\left\{1+\mathbf{r} \partial / \partial \mathbf{r}_{c}\right\} \mathcal{K}_{P}\left(\mathbf{r}_{c}\right)$, which reproduces the isotropic but not the anisotropic contribution in Eq. (6.1).

The local polymer pressure $p$ along the surface of $S$ in the presence of $P$ follows from Eq. (6.1), with $\mathcal{K}=\mathcal{M}$, via the density-pressure relation

$$
\mathcal{M}_{S, P}=B_{g}\left[(r-R) / \mathcal{R}_{g}\right]^{1 / \nu} p /\left(k_{B} T n\right), \quad l \ll r-R \ll R .
$$

Here $l$ denotes microscopic lengths, $k_{B} T n$ is the bulk pressure in the dilute polymer solution, where $n$ is the density of polymer chains, and $B_{g}$ is a universal number. ${ }^{13}$ The same number appears in the near wall behavior $\mathcal{M}_{\mathrm{hs}}=B_{g}\left(z / \mathcal{R}_{g}\right)^{1 / \nu}$ of the profile $\mathcal{M}=\mathcal{M}_{\mathrm{hs}}$ in a half space without particles, where the pressure onto the wall is the bulk pressure $k_{B} T n$. Here $z$ is the distance from the wall with $l \ll z \ll \mathcal{R}_{g}$.

Using Eqs. (2.2) and (3.4) to calculate $\mathcal{A}$ and $\tilde{\mathcal{A}}$ for $y \rightarrow 0$, Eqs. (6.1) and (6.4) yield

$$
\frac{p}{k_{B} T n}=A_{g}\left(\frac{\mathcal{R}_{g}}{R}\right)^{1 / \nu} \frac{1}{\Omega_{d}}\left\{x_{\epsilon}+d \mathbf{r} \frac{\partial}{\partial \mathbf{r}_{c}}\right\} \mathcal{M}_{P}\left(\mathbf{r}_{c}\right), \quad r=R,
$$

where $A_{g}$ is related to $B_{g}$ via

$$
A_{g} B_{g}=\frac{\left(A_{\epsilon}^{(\text {ord })}\right)^{2}}{B_{\epsilon}} 2^{-x_{\epsilon}} x_{\epsilon} C^{(\text {ord })} \text {. }
$$

Since $\mathcal{M}_{P}$ increases on moving away from $P$, due to the depletion effect, the pressure is larger for the surface points on $S$ that are farther away from $P$, so that $S$ is pushed toward $P$.

It is instructive to check Eq. (6.5) by calculating the insertion free energy $F$ and the total force $\mathbf{f}$ on the particle $S$. 
The insertion free energy equals the work needed to expand the particle from a point with radius $\hat{R}=0$ to its actual size $\hat{R}=R$ and is given by

$$
F=\int_{\hat{R}=0}^{\hat{R}=R} \mathrm{~d} \hat{F}, \quad \mathrm{~d} \hat{F}=\mathrm{d} \hat{R} \int \mathrm{d}|\hat{\mathbf{S}}| p(\hat{R}) .
$$

Only the first term in curly brackets of Eq. (6.5) contributes to the integral and yields

$$
\frac{F}{k_{B} T n}=A_{g} \mathcal{R}_{g}^{1 / \nu} \mathcal{M}_{P}\left(\mathbf{r}_{c}\right) I,
$$

where

$$
I=x_{\epsilon} \int_{0}^{R} \mathrm{~d} \hat{R}(\hat{R})^{d-1-(1 / \nu)}=R^{d-(1 / \nu)},
$$

since $x_{\epsilon}=d-(1 / \nu)$. The total force on the particle $S$ per $k_{B} T$ is given by Eq. (3.8), with $\tau_{S, P}$ replaced by $-p /\left(k_{B} T\right)$, so that only the second term in curly brackets of Eq. (6.5) contributes and yields a force

$$
\frac{\mathbf{f}}{k_{B} T n}=-A_{g} \mathcal{R}_{g}^{1 / \nu} R^{d-(1 / \nu)} \frac{\partial \mathcal{M}_{P}\left(\mathbf{r}_{c}\right)}{\partial \mathbf{r}_{c}},
$$

which pushes the particle $S$ toward the perturbing wall or particle $P$. Equations (6.8)-(6.10) are consistent with each other and with known results, see Eq. (6.7) in the first reference of Ref. 13.

We mention two special cases for the density profiles in Eq. (6.1):

(i) For a planar wall $P$ we may use the half-space geometry just above Eq. (3.9) and shown in Fig. 1, for which Eq. (6.1) yields

$\mathcal{K}_{S, \mathrm{hs}}(\mathbf{r}) \rightarrow\left\{\mathcal{A}_{\psi}^{(\psi, \text { ord })}-\widetilde{\mathcal{A}}_{\psi}^{(\psi, \text { ord })} r_{\perp} \frac{\mathrm{d}}{\mathrm{d} \mathcal{D}}\right\} \mathcal{K}_{\mathrm{hs}}\left(\mathcal{D} / \mathcal{R}_{g}\right)$,

where the bulk-normalized densities $\mathcal{K}_{\mathrm{hs}}$ in the half space only depend on the ratio of the distance $\mathcal{D}$ from its repulsive boundary wall and $\mathcal{R}_{g}$.

(ii) For a nanosphere $P$ with center at $\mathbf{r}_{c}+\mathbf{r}_{P}$ and a radius $R_{P}$ such that $R_{P}, R, r \ll r_{P}, \mathcal{R}_{g}$, one may evaluate $\mathcal{K}_{P}\left(\mathbf{r}_{c}\right)$ on the right-hand side of Eq. (6.1) using the small sphere expansion (2.8) for $P$. This yields

$$
\begin{aligned}
\lim _{\mathcal{R}_{g} \rightarrow \infty} \mathcal{K}_{S, \text { sph }}(\mathbf{r}) \rightarrow & \mathcal{A}_{\psi}^{(\psi, \text { ord })}-R_{P}^{x_{\epsilon}} \frac{A_{\epsilon}^{(\text {ord })}}{B_{\epsilon}^{1 / 2}}\left|\frac{C_{\epsilon \psi \psi}}{B_{\epsilon}^{1 / 2} B_{\psi}}\right| \\
& \times\left\{\frac{\mathcal{A}_{\psi}^{(\psi, \text { ord })}}{r_{P}^{x_{\epsilon}}}+x_{\epsilon} \widetilde{\mathcal{A}}_{\psi}^{(\psi, \text { ord })} \frac{\mathbf{r r}_{P}}{r_{P}^{x_{\epsilon}+2}}\right\}
\end{aligned}
$$

in $^{32}$ the limit $\mathcal{R}_{g} \rightarrow \infty$ and for $\mathbf{r}_{c}=\mathbf{0}$. For ideal chains, Eq. (6.12) is consistent with the behavior near one of the two spheres of the density $\mathcal{E}_{s s, \text { id }}$ given in Eqs. (3) ff. in Ref. 10, see in particular the derivatives of $\mathcal{E}$ at the sphere surface given in the first two lines below Fig. 2. For self-repelling chains and $d=2$, the right-hand side of Eq. (6.12) could be evaluated for $(\mathcal{K}=\mathcal{M}, \psi=\epsilon)$, since the negative quantity between vertical bars then equals the $N \rightarrow 0$ limit of $B_{2}^{\text {(ord) }}=1 / T_{21}$ in Ref. 29 , and $\mathcal{A}_{\epsilon}^{(\epsilon \text {,ord })}$ and $\widetilde{\mathcal{A}}_{\epsilon}^{(\epsilon, \text { ord })}$ are determined by Eq. (6.3).

Equations (6.4), (6.5), and (6.10) for nonadsorbing polymers are the counterparts of Eqs. (3.6), (3.7), and (3.2) for a binary liquid mixture or the Ising model. Comparing Eq. (3.2) at the critical point with Eq. (6.10) shows that in both cases the interaction induced between $S$ and $P$ with equal surface classes $a=b$ is attractive. Obviously there remain important differences such as the quantity $n \mathcal{R}_{g}^{1 / \nu}$ in Eq. (6.10), which is proportional to the polymer segment density in the bulk solution and does not appear in Eq. (3.2). One example is the interaction free energy per $k_{B} T$ of two nanoparticles $S$, $P$ with the quite different scaling-dimensionless forms $-\zeta_{p}\left(R R_{P}\right)^{x_{\epsilon}} n \mathcal{R}_{g}^{1 / \nu} / r_{P}^{x_{\epsilon}}$ and $-\zeta_{i}\left(R R_{P}\right)^{x_{\epsilon}} / r_{P}^{2 x_{\epsilon}}$ for nonadsorbing polymers $^{21}$ with $\mathcal{R}_{g} \rightarrow \infty$ and for "ordinary" surfaces in the Ising model at the critical point, ${ }^{3,17}$ respectively. In the notation introduced above the universal prefactors read $\zeta_{p}=A_{g} A_{\epsilon}^{(\text {ord })}\left|C_{\epsilon \epsilon \epsilon}\right| / B_{\epsilon}^{2}$ and $\zeta_{i}=\left(A_{\epsilon}^{(\text {ord })}\right)^{2} / B_{\epsilon}$.

\section{SUMMARY AND CONCLUDING REMARKS}

We have shown how to evaluate the effect of distant perturbations on the density profiles $\langle\psi\rangle$ near a small mesoscopic inclusion or particle $S$ of spherical shape in a critical system. On large length scales of the correlation length $\xi$ and the distance $\mathcal{D}$ between $S$ and the perturbation, the combination $(S, \psi)$ of $S$ and the nearby density operator $\psi$ appear as a small object that can be expanded in terms of bulk operators multiplied by amplitudes. ${ }^{33}$ The leading isotropic and anisotropic operators are the order parameter $\phi$ and the energy density $\epsilon$ and their first derivatives. The amplitudes depend on the radius $R$ of $S$ and the distance $r$ of $\psi$ from the center of $S$ and are determined by two-point cumulants of $\phi$ and $\epsilon$ in the half space (or, equivalently, in the space outside the particle $S$ ), right at the critical point, as given in Eqs. (2.1)-(2.4). This allows us to calculate the perturbationinduced density corrections that are isotropic and anisotropic about the center of $S$.

We have checked this type of "fusion" in several ways. In Sec. III we show that in the special cases $r \gg R$ and $r-R \ll R$ it reduces to the well established small sphere ${ }^{16,17}$ and boundary operator ${ }^{9}$ expansions, respectively. The density corrections in the latter case are proportional to the local pressure induced at the surface of the spherical particle.

If the system is right at the critical point and the distant perturbation $P$ is a sphere or a planar wall, one can derive the fusion for arbitrary $r / R$ by applying the small sphere expansion to a conformal image of $P$, as shown in Sec. IV.

Besides expression (2.6) for the density corrections, we have also checked the consequences (2.7) of the fusion for two and many-point correlation functions, see Sec. II A and Appendix A.

This approach allows us to predict the density corrections in cases where the two-point functions in the half space are known. In Sec. V we obtain explicit results for the isotropic and anisotropic corrections near "ordinary" and "nor- 
mal" spheres $S$ due to a distant planar wall in the critical Gaussian and Ising models. The latter is relevant for a critical binary liquid mixture. ${ }^{12}$ In Sec. VI we present the results for the density corrections in a solution of long, flexible, nonadsorbing polymers.

It would be interesting to compare the predictions with simulations for polymer systems and further analytic work for the Ising model. ${ }^{34}$

Considering an $m$-point function instead of the density profile near the sphere, we expect that the effect of distant perturbations can be described by a fusion of the sphere with the $m$ operators.

\section{ACKNOWLEDGMENTS} cussions.

It is a pleasure to thank T. W. Burkhardt for useful dis-

\section{APPENDIX A: MANY-POINT AVERAGES}

Here we present further instructive checks of the fusion relation (2.7).

\section{1. "Ordinary" surfaces in the Gaussian model}

The Gaussian model in $d>2$ with ordinary surfaces is characterized by the Hamiltonian

$$
H=\frac{1}{2} \int \mathrm{d} \mathbf{r}\left[(\nabla \phi(\mathbf{r}))^{2}+t(\phi(\mathbf{r}))^{2}\right],
$$

where the integral extends over the space bounded by the surfaces and $\phi$ vanishes at the surfaces. ${ }^{9}$ The non-negative parameter $t$ is proportional to $\xi^{-2}$ and vanishes at the critical point.

Here we consider the critical system with $t=0$ enclosed in a spherical container $P$ of radius $R_{+}$, which has the same center $\mathbf{r}_{c}$ as the sphere $S$. First we check Eq. (2.7) for $n=1$ and $\mathcal{O}_{1}=\psi=\phi$. With $\mathbf{r}_{c}=\mathbf{0}$ and $R<r<r_{1}<R_{+}$, one finds ${ }^{18}$

$$
\begin{aligned}
\left\langle\phi\left(\mathbf{r}_{1}\right) \phi(\mathbf{r})\right\rangle_{S, P} / B_{\phi}= & \sum_{l=0}^{\infty} C_{l}^{(\alpha)}(\cos \vartheta)\left(r_{1} r\right)^{l}\left(r_{1}^{-\ell}-R_{+}^{-\ell}\right) \\
& \times\left[1-(R / r)^{\ell}\right]\left\{1-\left(R / R_{+}\right)^{\ell}\right\}^{-1}
\end{aligned}
$$

with notation from Eq. (2.11) and $\ell=2(\alpha+l)$. The leading isotropic and anisotropic terms for $R, r \ll r_{1}, R_{+}$correspond to $l=0$ and $l=1$, respectively, and are given by

$$
\begin{aligned}
& \left\langle\phi\left(\mathbf{r}_{1}\right) \phi(\mathbf{r})\right\rangle_{S, P} /\left.B_{\phi}\right|_{\text {iso }}=\left(r_{1}^{-(d-2)}-R_{+}^{-(d-2)}\right) \mathcal{A}_{\phi}^{(\phi, \text { ord })}, \\
& \left\langle\phi\left(\mathbf{r}_{1}\right) \phi(\mathbf{r})\right\rangle_{S, P} /\left.B_{\phi}\right|_{\text {aniso }}=(d-2) \mathbf{r}_{1} \mathbf{r}\left(r_{1}^{-d}-R_{+}^{-d}\right) \tilde{\mathcal{A}}_{\phi}^{(\phi, \text { ord })},
\end{aligned}
$$

with $\mathcal{A}$ and $\tilde{\mathcal{A}}$ from Eq. (2.15). Comparing Eqs. (A3) and (A4) with the expressions

$$
\begin{aligned}
& \left\langle\phi\left(\mathbf{r}_{1}\right) \phi(\mathbf{0})\right\rangle_{P} / B_{\phi}=r_{1}^{-(d-2)}-R_{+}^{-(d-2)}, \\
& \left(\partial / \partial \mathbf{r}_{c}\right)\left\langle\phi\left(\mathbf{r}_{1}\right) \phi\left(\mathbf{r}_{c}\right)\right\rangle_{P} /\left.B_{\phi}\right|_{r_{c}=0}=(d-2) \mathbf{r}_{1}\left(r_{1}^{-d}-R_{+}^{-d}\right)
\end{aligned}
$$

that follow from the propagator inside the container $P$ with center at the origin,

$$
\begin{aligned}
\left\langle\phi\left(\mathbf{r}_{1}\right) \phi\left(\mathbf{r}_{c}\right)\right\rangle_{P} / B_{\phi}= & {\left[r_{1}^{2}+r_{c}^{2}-2 \mathbf{r}_{1} \mathbf{r}_{c}\right]^{-\alpha} } \\
& -\left[R_{+}^{2}+\left(r_{1} r_{c} / R_{+}\right)^{2}-2 \mathbf{r}_{1} \mathbf{r}_{c}\right]^{-\alpha}
\end{aligned}
$$

confirms Eq. (2.7) for $\mathcal{O}_{1}=\psi=\phi$. This has the form of the first and last expressions in Eq. (2.14), with the subscripts $S$ and "bulk" replaced by $S, P$, and $P$, respectively. The validity for $\mathcal{O}_{1}=\psi=\epsilon$ follows from the same steps that led from Eq. (2.14) to Eq. (2.18).

Now consider Eq. (2.7) for $n=2$ and $\mathcal{O}_{1}=\mathcal{O}_{2}=\psi=\epsilon$. The expression

$$
\begin{aligned}
\left\langle\epsilon\left(\mathbf{r}_{1}\right)\right. & \left.\boldsymbol{\epsilon}\left(\mathbf{r}_{2}\right) \boldsymbol{\epsilon}(\mathbf{r})\right\rangle_{S, P}-\left\langle\epsilon\left(\mathbf{r}_{1}\right) \epsilon\left(\mathbf{r}_{2}\right)\right\rangle_{S, P}\langle\epsilon(\mathbf{r})\rangle_{S, P} \\
= & 2\left[\left\langle\boldsymbol{\epsilon}\left(\mathbf{r}_{1}\right)\right\rangle_{S, P}\left(\left\langle\phi\left(\mathbf{r}_{2}\right) \phi(\mathbf{r})\right\rangle_{S, P}\right)^{2}+(1 \leftrightarrow 2)\right] B_{\epsilon} / B_{\phi^{2}} \\
& -8\left\langle\phi\left(\mathbf{r}_{1}\right) \phi\left(\mathbf{r}_{2}\right)\right\rangle_{S, P}\left\langle\phi\left(\mathbf{r}_{1}\right) \phi(\mathbf{r})\right\rangle_{S, P}\left\langle\phi\left(\mathbf{r}_{2}\right) \phi(\mathbf{r})\right\rangle_{S, P} \\
& \times\left(B_{\epsilon} / B_{\phi^{2}}\right)^{3 / 2}
\end{aligned}
$$

for the left-hand side follows from $\epsilon=-\left(B_{\epsilon} / B_{\phi^{2}}\right)^{1 / 2} \phi^{2}$, dimensional regularization, and Wick's theorem. The leading behavior of $\left\langle\phi\left(\mathbf{r}_{i}\right) \phi(\mathbf{r})\right\rangle_{S, P}$ with $i=1,2$ has been discussed above. For $\left\langle\epsilon\left(\mathbf{r}_{i}\right)\right\rangle_{S, P}$ and $\left\langle\phi\left(\mathbf{r}_{1}\right) \phi\left(\mathbf{r}_{2}\right)\right\rangle_{S, P}$ the small sphere expansion for $S$ in Eq. (2.8) implies the leading behaviors $\left\langle\epsilon\left(\mathbf{r}_{i}\right)\right\rangle_{P}$ and $\left\langle\phi\left(\mathbf{r}_{1}\right) \phi\left(\mathbf{r}_{2}\right)\right\rangle_{P}$, respectively. Thus, the leading behavior of Eq. (A8) is indeed described by Eq. (2.7), i.e., by the expression in curly brackets in Eq. (2.18) acting upon the right-hand side of Eq. (A8), where $S, P$ is replaced by $P, \mathbf{r}$ is replaced by $\mathbf{r}_{c}$, and $\mathbf{r}_{c}$ is set equal to $\mathbf{0}$ after the differentiation.

Similar arguments apply to the case $n=2, \mathcal{O}_{1}=\mathcal{O}_{2}=\phi$, and $\psi=\epsilon$, in which

$$
\begin{aligned}
& \left\langle\phi\left(\mathbf{r}_{1}\right) \phi\left(\mathbf{r}_{2}\right) \boldsymbol{\epsilon}(\mathbf{r})\right\rangle_{S, P}-\left\langle\phi\left(\mathbf{r}_{1}\right) \phi\left(\mathbf{r}_{2}\right)\right\rangle_{S, P}\langle\boldsymbol{\epsilon}(\mathbf{r})\rangle_{S, P} \\
& \quad=-2\left\langle\phi\left(\mathbf{r}_{1}\right) \phi(\mathbf{r})\right\rangle_{S, P}\left\langle\phi\left(\mathbf{r}_{2}\right) \phi(\mathbf{r})\right\rangle_{S, P}\left(B_{\epsilon} / B_{\phi^{2}}\right)^{1 / 2} .
\end{aligned}
$$

This case is related to the monomer density in a solution of ideal polymer chains which are described by the Gaussian model. $^{28}$

\section{2. "Normal" sphere in the critical $d=2$ Ising model}

Here we check the fusion relation (2.7) for a circular disk $S$ with a normal boundary $a=+$ in the $d=2$ Ising model right at the critical point. We consider the case where $P$ is absent and $\mathcal{O}_{1}=\ldots=\mathcal{O}_{n}=\psi=\phi$ and compare it with the exact result

$$
\begin{aligned}
&\left\langle\phi\left(\mathbf{r}_{1}\right) \ldots \phi\left(\mathbf{r}_{n}\right) \phi(\mathbf{r})\right\rangle_{S} / B_{\phi}^{(n+1) / 2}\left(\frac{R}{r_{1}^{2}-R^{2}} \ldots \frac{R}{r_{n}^{2}-R^{2}} \frac{1}{R y}\right)^{1 / 8} \\
& \quad \times\left\{2^{-(n+1) / 2} \sum_{\mu_{1}= \pm 1} \ldots \sum_{\mu_{n}= \pm 1} \sum_{\mu_{n+1}= \pm 1} \Pi_{i<j}\left(\chi_{i j}\right)^{\mu_{i} \mu_{j}}\right\}^{1 / 2}
\end{aligned}
$$

in the plane outside the circular disk $S$ centered at $\mathbf{r}_{c}=\mathbf{0}$. Here $y$ is from Eq. (2.3) and

$$
\chi_{i j}=\left[1+\frac{\left(r_{i}^{2}-R^{2}\right)\left(r_{j}^{2}-R^{2}\right)}{r_{i j}^{2} R^{2}}\right]^{-1 / 4},
$$




$$
r_{i j} \equiv\left|\mathbf{r}_{i}-\mathbf{r}_{j}\right|, \quad \mathbf{r}_{n+1} \equiv \mathbf{r} .
$$

Equations (A10) and (A11) follow via a conformal transformation ${ }^{20}$ from the corresponding multipoint average in the half plane in Eq. (9a) of Ref. 35.

First we discuss Eq. (2.7) for $n=2$. Here Eq. (A10) implies

$$
\begin{aligned}
& \left\langle\phi\left(\mathbf{r}_{1}\right) \phi\left(\mathbf{r}_{2}\right) \phi(\mathbf{r})\right\rangle_{S} / B_{\phi}^{3 / 2} \\
& \quad \rightarrow r_{12}^{-1 / 4} \Phi_{S}+\left.\left\{f_{\epsilon}+\tilde{\mathcal{A}}_{\epsilon}^{(\phi,+)} \mathbf{r} \frac{\partial}{\partial \mathbf{r}_{c}}\right\} U\right|_{r_{c}=0}+\mathcal{A}_{T}^{(\phi,+)} V
\end{aligned}
$$

and

$$
\left\langle\phi\left(\mathbf{r}_{1}\right) \phi\left(\mathbf{r}_{2}\right)\right\rangle_{S}\langle\phi(\mathbf{r})\rangle_{S} / B_{\phi}^{3 / 2} \rightarrow r_{12}^{-1 / 4} \Phi_{S}+\left.g_{\epsilon} U\right|_{r_{c}=0},
$$

where

$$
\begin{aligned}
\Phi_{S} \equiv\langle\phi(\mathbf{r})\rangle_{S} / B_{\phi}^{1 / 2}= & 2^{1 / 4}(R y)^{-1 / 8}, \\
\left\{f_{\epsilon}, g_{\epsilon} ; \widetilde{\mathcal{A}}_{\epsilon}^{(\phi,+)}, \mathcal{A}_{T}^{(\phi,+)}\right\}= & -\Phi_{S}\left\{R \frac{1+(y / 2)}{(1+y)^{1 / 2}}, R ;\right. \\
& \left.R \frac{y^{2}}{4(1+y)^{3 / 2}}, \frac{\pi}{2} \frac{y^{2}}{(1+y)^{2}}\right\},
\end{aligned}
$$

and

$$
\begin{aligned}
U \equiv\left\langle\phi\left(\mathbf{r}_{1}\right) \phi\left(\mathbf{r}_{2}\right) \epsilon\left(\mathbf{r}_{c}\right)\right\rangle_{\mathrm{bulk}} /\left(B_{\phi} B_{\epsilon}^{1 / 2}\right), \\
\left.\left(1, \mathbf{r} \frac{\partial}{\partial \mathbf{r}_{c}}\right) U\right|_{r_{c}=0}=-\frac{r_{12}^{3 / 4}}{2 r_{1} r_{2}}\left(1, \frac{\mathbf{r r}_{1}}{r_{1}^{2}}+\frac{\mathbf{r r}_{2}}{r_{2}^{2}}\right), \\
V \equiv\left\langle\phi\left(\mathbf{r}_{1}\right) \phi\left(\mathbf{r}_{2}\right) \sum_{k, l} r_{k} r_{l} T_{k l}(0)\right\rangle_{\text {bulk }} / B_{\phi} \\
=-\frac{1}{8 \pi} r_{12}^{-1 / 4}\left[\frac{\left(\mathbf{r}_{1} \mathbf{r}\right)^{2}}{r_{1}^{4}}+\frac{\left(\mathbf{r}_{2} \mathbf{r}\right)^{2}}{r_{2}^{4}}-2 \frac{\left(\mathbf{r}_{1} \mathbf{r}\right)\left(\mathbf{r}_{2} \mathbf{r}\right)}{\left(r_{1} r_{2}\right)^{2}}\right. \\
\left.\quad-\frac{r^{2}}{2}\left(\frac{r_{12}}{r_{1} r_{2}}\right)^{2}\right] .
\end{aligned}
$$

Terms of order 3-(1/8) in the small lengths $R$ and $r$ have been suppressed on the right-hand side of Eq. (A12). The last term on the right-hand side of Eq. (A12) requires augmenting $\mathcal{F}^{(\phi,+)}$ in Eq. (2.1) by a term $\mathcal{A}_{T}^{(\phi,+)} \Sigma_{k, l} r_{k} r_{l} T_{k l}\left(\mathbf{r}_{c}\right)$, where $T_{k l}$ is the stress tensor, see the discussion between Eqs. (2.4) and (2.5). In the $d=2$ Ising model both the stress tensor and the first derivative of the energy density have scaling dimension 2 and in Eq. (A12) they lead to terms of order 2 $-(1 / 8)$ in the small lengths. These terms are even and odd in $\mathbf{r}$, respectively. Equations (A12)-(A15) are consistent with Eq. (2.7) since $\mathcal{A}_{\epsilon}^{(\phi,+)}$ equals $f_{\epsilon}-g_{\epsilon}, \chi=\phi$ does not contribute in $\left\langle\phi\left(\mathbf{r}_{1}\right) \phi\left(\mathbf{r}_{2}\right) \chi\left(\mathbf{r}_{c}\right)\right\rangle_{\text {bulk }}$, and $\langle\mathcal{F}\rangle_{\text {bulk }}$ and $\left\langle T_{k l}\right\rangle_{\text {bulk }}$ vanish. We have checked that the amplitude $\mathcal{A}_{T}^{(\phi,+)}$ of Eq. (A14) also appears in the disk average

$$
\left\langle T_{\kappa \lambda}\left(\mathbf{r}_{1}\right) \phi(\mathbf{r})\right\rangle_{S} / B_{\phi}^{1 / 2} \rightarrow \mathcal{A}_{T}^{(\phi,+)}\left\langle T_{\kappa \lambda}\left(\mathbf{r}_{1}\right) \sum_{k, l} r_{k} r_{l} T_{k l}(0)\right\rangle_{\text {bulk }},
$$

Now we check Eq. (2.7) for $n=3$. From Eq. (A10),

$$
\left\langle\phi\left(\mathbf{r}_{1}\right) \phi\left(\mathbf{r}_{2}\right) \phi\left(\mathbf{r}_{3}\right) \phi(\mathbf{r})\right\rangle_{S} /\left.B_{\phi}^{2} \rightarrow\left\{f_{\phi}+\tilde{\mathcal{A}}_{\phi}^{(\phi,+)} \mathbf{r} \frac{\partial}{\partial \mathbf{r}_{c}}\right\} W\right|_{r_{c}=0}
$$

and

$$
\left\langle\phi\left(\mathbf{r}_{1}\right) \phi\left(\mathbf{r}_{2}\right) \phi\left(\mathbf{r}_{3}\right)\right\rangle_{S}\langle\phi(\mathbf{r})\rangle_{S} /\left.B_{\phi}^{2} \rightarrow g_{\phi} W\right|_{r_{c}=0},
$$

where

$$
\left\{f_{\phi}, g_{\phi} ; \tilde{\mathcal{A}}_{\phi}^{(\phi,+)}\right\}=y^{-1 / 8}\left\{p_{+}^{1 / 2}, \sqrt{2} ; \frac{y}{1+y} \frac{p_{-}}{p_{+}^{1 / 2}}\right\},
$$

$$
p_{ \pm}=(1+y)^{1 / 4} \pm(1+y)^{-1 / 4},
$$

and

$$
\begin{aligned}
W \equiv & \left\langle\phi\left(\mathbf{r}_{1}\right) \phi\left(\mathbf{r}_{2}\right) \phi\left(\mathbf{r}_{3}\right) \phi\left(\mathbf{r}_{c}\right)\right\rangle_{\text {bulk }} / B_{\phi}^{2} \\
= & 2^{-1 / 2}\left[\left(\frac{r_{12}}{r_{13} r_{23}} \frac{r_{3 c}}{r_{1 c} r_{2 c}}\right)^{1 / 2}+\left(\frac{r_{13}}{r_{12} r_{23}} \frac{r_{2 c}}{r_{1 c} r_{3 c}}\right)^{1 / 2}\right. \\
& \left.+\left(\frac{r_{23}}{r_{12} r_{13}} \frac{r_{1 c}}{r_{2 c} r_{3 c}}\right)^{1 / 2}\right]^{1 / 2},
\end{aligned}
$$

with $r_{1 c} \equiv\left|\mathbf{r}_{1}-\mathbf{r}_{c}\right|$, etc. This is consistent with Eq. (2.7) since $\mathcal{A}_{\phi}^{(\phi,+)}$ equals $f_{\phi}-g_{\phi}$ and since $\chi=\epsilon$ or $\chi=T_{k l}$ do not contribute in $\left\langle\phi\left(\mathbf{r}_{1}\right) \phi\left(\mathbf{r}_{2}\right) \phi\left(\mathbf{r}_{3}\right) \chi\left(\mathbf{r}_{c}\right)\right\rangle_{\text {bulk }}$.

\section{APPENDIX B: OPERATOR PRODUCT EXPANSIONS}

The expansions needed in Sec. III B are

$$
\begin{aligned}
\psi\left(\mathbf{r}_{c}\right) \psi\left(\mathbf{r}_{c}+\mathbf{r}\right)-\frac{B_{\psi}}{r^{2 x_{\psi}} \rightarrow} & \frac{C_{\psi \psi \epsilon}}{B_{\epsilon}} r^{x_{\epsilon}-2 x_{\psi}}\left[1+\frac{\mathbf{r}}{2} \frac{\partial}{\partial \mathbf{r}_{c}}\right] \epsilon\left(\mathbf{r}_{c}\right) \\
& +\ldots, \\
\phi\left(\mathbf{r}_{c}\right) \epsilon\left(\mathbf{r}_{c}+\mathbf{r}\right) \rightarrow & \frac{C_{\phi \phi \epsilon}}{B_{\phi}} r^{-x_{\epsilon}}\left[1+\frac{x_{\epsilon}}{2 x_{\phi}} \mathbf{r} \frac{\partial}{\partial \mathbf{r}_{c}}\right] \phi\left(\mathbf{r}_{c}\right)+\ldots, \\
\epsilon\left(\mathbf{r}_{c}\right) \phi\left(\mathbf{r}_{c}+\mathbf{r}\right) \rightarrow & \frac{C_{\phi \phi \epsilon}}{B_{\phi}} r^{-x_{\epsilon}}\left[1+\frac{2 x_{\phi}-x_{\epsilon}}{2 x_{\phi}} \mathbf{r} \frac{\partial}{\partial \mathbf{r}_{c}}\right] \phi\left(\mathbf{r}_{c}\right) \\
& +\ldots,
\end{aligned}
$$

where the ellipses denote contributions from second and higher derivatives of $\epsilon$ and $\phi$ and from higher operators. For more details see, e.g., Eqs. (2.39) and (2.47) of Ref. 25 and references therein.

\section{APPENDIX C: CONFORMAL TRANSFORMATION}

Solving the special conformal transformation (4.1) for $\mathbf{r}^{\prime}$ and $\mathbf{r}$ yields

$$
\mathbf{r}^{\prime}=b\left(\mathbf{r}^{\prime}\right)\left\{-\frac{\mathbf{R}}{2}\left(\frac{r^{2}}{R^{2}}-1\right)+\mathbf{r}-\frac{\mathbf{R}(\mathbf{R} \mathbf{r})}{R^{2}}\right\},
$$

where $T_{\kappa \lambda}$ is a distant stress tensor. 


$$
\mathbf{r}=\frac{1}{b\left(\mathbf{r}^{\prime}\right)}\left\{\mathbf{R}\left(\frac{r^{\prime 2}}{4 R^{2}}-1\right)+\mathbf{r}^{\prime}-\frac{\mathbf{R}\left(\mathbf{R r}^{\prime}\right)}{R^{2}}\right\} .
$$

Here

$$
b\left(\mathbf{r}^{\prime}\right)=\left|\partial \mathbf{r} / \partial \mathbf{r}^{\prime}\right|^{-1 / d}=\frac{4 R^{2}}{|\mathbf{r}-\mathbf{R}|^{2}}=\frac{\left|\mathbf{r}^{\prime}+2 \mathbf{R}\right|^{2}}{4 R^{2}}
$$

is the local dilatation, which also enters the relation ${ }^{14}$

$$
\begin{aligned}
& \left\langle\psi_{1}\left(\mathbf{r}_{1}\right) \ldots \psi_{N}\left(\mathbf{r}_{N}\right)\right\rangle_{G} \\
& \quad=\left[b\left(\mathbf{r}_{1}^{\prime}\right)\right]^{x_{\psi_{1}} \ldots[}\left[b\left(\mathbf{r}_{N}^{\prime}\right)\right]^{x_{\psi_{N}}\left\langle\psi_{1}\left(\mathbf{r}_{1}^{\prime}\right) \ldots \psi_{N}\left(\mathbf{r}_{N}^{\prime}\right)\right\rangle_{G^{\prime}}}
\end{aligned}
$$

between averages in the original and transformed boundary geometries $G$ and $G^{\prime}$, respectively.

The identities

$$
\frac{b\left(\mathbf{r}_{1}^{\prime}\right) b\left(\mathbf{r}_{2}^{\prime}\right)}{\left|\mathbf{r}_{1}^{\prime}-\mathbf{r}_{2}^{\prime}\right|^{2}}=\frac{1}{\left|\mathbf{r}_{1}-\mathbf{r}_{2}\right|^{2}}
$$

and

$$
\frac{b\left(\mathbf{r}^{\prime}\right)}{2\left|\mathbf{r}^{\prime} \mathbf{R} / R\right|}=\frac{R}{r^{2}-R^{2}}
$$

follow from Eqs. (C1) and (C2) and are consistent, respectively, with the invariant form of the two-point function $\langle\psi \psi\rangle_{\text {bulk }}$ in a bulk system without boundaries under special conformal transformations and with ${ }^{20}$ the form $A_{\psi}^{(a)}\left(R /\left(r^{2}-R^{2}\right)\right)^{x_{\psi}}$ of the unperturbed density profile $\langle\psi\rangle_{S}$ around $S$ at the critical point.

\section{APPENDIX D: POLYMER DENSITIES}

Here we derive Eq. (6.1). The density $\mathcal{M}_{S, P}$ at $\mathbf{r}_{c}+\mathbf{r}$ in a dilute solution equals the normalized partition function

$$
\mathcal{M}_{S, P}\left(\mathbf{r}_{c}+\mathbf{r}\right)=M_{S, P}\left(\mathbf{r}_{c}+\mathbf{r}\right) / M_{\text {bulk }}
$$

of a single chain with any segment at $\mathbf{r}_{c}+\mathbf{r}$. The numerator $M_{S, P}$ is given by

$$
\begin{aligned}
M_{G}\left(\mathbf{r}_{c}+\mathbf{r}\right)= & -\mathcal{L} \int_{G} \mathrm{~d} \mathbf{r}_{1} \int_{G} \mathrm{~d} \mathbf{r}_{2}\left[\left\langle\phi\left(\mathbf{r}_{1}\right) \phi\left(\mathbf{r}_{2}\right) \epsilon\left(\mathbf{r}_{c}+\mathbf{r}\right)\right\rangle_{G}\right. \\
& \left.-\left\langle\phi\left(\mathbf{r}_{1}\right) \phi\left(\mathbf{r}_{2}\right)\right\rangle_{G}\left\langle\epsilon\left(\mathbf{r}_{c}+\mathbf{r}\right)\right\rangle_{G}\right]
\end{aligned}
$$

with $G=S, P$, and the denominator $M_{\text {bulk }}$ equals Eq. (D2), where $G$ denotes the unbounded bulk space. In Eq. (D2), $\mathcal{L}$ denotes a (inverse) Laplace transform that relates the molecular weight of the polymer chain to the temperature deviation from $T_{c}$ in the high temperature phase of the Gaussian or $N \rightarrow 0$ vector models. ${ }^{13,28}$

Using the fusion relation (2.7) with $n=2, \mathcal{O}_{1}=\mathcal{O}_{2}=\phi$, and $(\psi, a)=(\epsilon$, ord $)$, and taking into account that the contributions of the regions $\left|\mathbf{r}_{i}-\mathbf{r}_{c}\right|$ of order $R$ are negligible for $R \ll \mathcal{D}, \mathcal{R}_{g}$ yields

$$
M_{S, P}\left(\mathbf{r}_{c}+\mathbf{r}\right) \rightarrow\left\{\mathcal{A}_{\epsilon}^{(\epsilon, \mathrm{ord})}+\tilde{\mathcal{A}}_{\epsilon}^{(\epsilon, \mathrm{ord})} \mathbf{r} \frac{\partial}{\partial \mathbf{r}_{c}}\right\} M_{P}\left(\mathbf{r}_{c}\right),
$$

where $M_{P}$ equals $M_{G}$ in Eq. (D2) with $G=P$. Equations (D1) and (D3) imply Eq. (6.1) for $\mathcal{K}=\mathcal{M}$. Equation (6.1) for $\mathcal{K}=\mathcal{E}$ is derived similarly by applying Eq. (2.7) with $n=1$,
$\mathcal{O}_{1}=\phi$, and $(\psi, a)=(\phi$, ord $)$ to the Laplace transform of the integral over $\mathbf{r}_{1}$ of $\left\langle\phi\left(\mathbf{r}_{1}\right) \phi\left(\mathbf{r}_{c}+\mathbf{r}\right)\right\rangle_{S, P}$.

${ }^{1}$ C. Hertlein, L. Helden, A. Gambassi, S. Dietrich, and C. Bechinger, Nature (London) 451, 172 (2008).

${ }^{2}$ M. E. Fisher and P. G. de Gennes, C. R. Acad. Sci. Paris B 287, 207 (1978).

${ }^{3}$ P. G. de Gennes, C. R. Acad. Sci. Paris II 292, 701 (1981).

${ }^{4}$ P. G. de Gennes, C. R. Acad. Sci. Paris B 288, 359 (1979).

${ }^{5}$ T. W. Burkhardt and T. Xue, Phys. Rev. Lett. 66, 895 (1991); T. W. Burkhardt and T. Xue, Nucl. Phys. B 354, 653 (1991).

${ }^{6}$ J. L. Cardy, Phys. Rev. Lett. 65, 1443 (1990).

${ }^{7}$ E. Eisenriegler, M. Krech, and S. Dietrich, Phys. Rev. Lett. 70, 619 (1993); 70, 2051 (1993).

${ }^{8}$ E. Eisenriegler and M. Stapper, Phys. Rev. B 50, 10009 (1994); E. Eisenriegler, M. Krech, and S. Dietrich, ibid. 53, 14377 (1996). In these two references the amplitude $C^{(a)}$ of Eq. (3.3) is denoted by $C_{\Psi} / x_{\Psi}$ and $C_{T} / x_{\Psi}$, respectively, and $T_{n n}$ is denoted by $T_{\perp \perp}$.

${ }^{9}$ H. W. Diehl, in Phase Transitions and Critical Phenomena, edited by C. Domb and J. L. Lebowitz (Academic, New York, 1986), Vol. 10; H. W. Diehl, Int. J. Mod. Phys. B 11, 3503 (1997). For earlier literature on boundary critical phenomena, see K. Binder, in Phase Transitions and Critical Phenomena, edited by C. Domb and J. L. Lebowitz (Academic, New York, 1983), Vol. 8.

${ }^{10}$ For the special case of the leading isotropic density correction for a nanoparticle with "ordinary" boundary condition, the fusion approach has been used before in E. Eisenriegler, J. Phys.: Condens. Matter 12, A227 (2000). Equation (2) of this reference corresponds to Eq. (2.7) in the absence of the distant perturbation $P$.

${ }^{11}$ G. Flöter and S. Dietrich, Z. Phys. B: Condens. Matter 97, 213 (1995); F. Schlesener, A. Hanke, and S. Dietrich, J. Stat. Phys. 110, 981 (2003); O. Vasilyev, A. Gambassi, A. Maciolek, and S. Dietrich, Europhys. Lett. 80, 60009 (2007).

${ }^{12}$ Due to universality the concentration profiles induced by boundaries and the corresponding interactions in a near critical binary liquid mixture follow (Refs. 1, 2, 9, and 11) from density profiles and thermodynamic Casimir forces in the Ising model with boundary magnetic fields.

${ }^{13}$ E. Eisenriegler, in Lecture Notes in Physics, edited by H. MeyerOrtmanns and A. Kluemper (Springer, New York, 1998), Vol. 508, pp. 1-24; E. Eisenriegler, in Soft Matter, edited by G. Gompper and M. Schick (Wiley, New York, 2005), Vol. 2.

${ }^{14}$ J. L. Cardy, in Phase Transitions and Critical Phenomena, edited by C. Domb and J. L. Lebowitz (Academic, New York, 1986), Vol. 11, p. 55.

${ }^{15}$ L. S. Brown, Ann. Phys. (N.Y.) 126, 135 (1980).

${ }^{16}$ The small sphere expansion applies if the sphere radius is much smaller than the correlation length and the distances from the sphere center to other surfaces and operators.

${ }^{17}$ T. W. Burkhardt and E. Eisenriegler, Phys. Rev. Lett. 74, 3189 (1995); 78, 2867 (1997).

${ }^{18}$ E. Eisenriegler and U. Ritschel, Phys. Rev. B 51, 13717 (1995).

${ }^{19}$ Neglecting the anomalous dimension $\eta$ in $x_{\phi}=(d-2+\eta) / 2$, results consistent with the predictions of the small sphere expansion (2.8) were obtained in Ref. 3 for the order-parameter density profile at a large distance from a single sphere and the interaction free energy of two distant spheres at the critical point of demixing.

${ }^{20}$ T. W. Burkhardt and E. Eisenriegler, J. Phys. A 18, L83 (1985). In this reference, $\mathbf{r}$ and $\mathbf{r}^{\prime}$ are denoted by $\mathbf{r}^{\prime \prime}$ and $\mathbf{r}$, respectively.

${ }^{21}$ A. Hanke, E. Eisenriegler, and S. Dietrich, Phys. Rev. E 59, 6853 (1999).

${ }^{22}$ In the Gaussian model, $x_{\epsilon}=2 x_{\phi}=d-2, \quad C_{\phi \phi \epsilon} /\left(B_{\phi} B_{\epsilon}^{1 / 2}\right)=-2^{1 / 2}$,

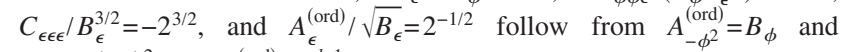
$B_{\phi^{2}}=2\left(B_{\phi}\right)^{2}$, and $C^{\text {(ord })}=2^{\epsilon-1} \Omega_{d}$ from Ref. 8 .

${ }^{23}-\tau_{S, P}$ equals the correction $\delta_{P}\left\langle T_{n n}\left(\mathbf{r}_{c}+R \mathbf{r} / r\right)\right\rangle_{S}$ to the stress tensor on the surface of the sphere $S$ and $-\tau_{W, P}$, the corresponding correction $\delta_{P}\left\langle T_{n n}\left(r_{\perp}=0, \mathbf{r}_{\|}\right)\right\rangle_{W}$ on the planar wall $W$. The two quantities equal the corrections to the pressure $/ k_{B} T$ on $S$ and $W$, respectively, due to the presence of $P$.

${ }^{24}$ For $0<R \ll r \ll \mathcal{D}$ and $(\psi, a)=(\epsilon, \pm)$, the profile correction $\delta_{P}\langle\epsilon\rangle_{S} / B_{\epsilon}^{1 / 2}$ tends to the sum of $\left[1+\mathbf{r} \partial /\left(\partial \mathbf{r}_{c}\right)\right] \delta_{P}\langle\epsilon\rangle_{\text {bulk }} / B_{\epsilon}^{1 / 2}$ and const $\left(R^{x_{\phi}} / r^{x}\right)[1$ $\left.+\left(x_{\epsilon} /\left(2 x_{\phi}\right)\right) \mathbf{r} \partial /\left(\partial \mathbf{r}_{c}\right)\right]\langle\phi\rangle_{P} / B_{\phi}^{1 / 2}$, where const is the constant multiplying $y^{x_{e} / 2}$ on the right-hand side of the second Eq. (3.14). While the leading behavior for $\mathcal{D} \rightarrow \infty$ with $0<R \ll r$ fixed is given by the second one of the two terms above, the limit $R \rightarrow 0$ with $r \ll \mathcal{D}$ fixed is given by the first term in accordance with Eq. (3.15). 
${ }^{25}$ E. Eisenriegler, J. Chem. Phys. 121, 3299 (2004).

${ }^{26}$ In the $d=2$ Ising model, $x_{\phi}=1 / 8, x_{\epsilon}=1, C_{\phi \phi \epsilon} /\left(B_{\phi} B_{\epsilon}^{1 / 2}\right)=-1 / 2, C_{\epsilon \epsilon \epsilon}=0$, $A_{\phi}^{(+)} / \sqrt{B_{\phi}}=2^{1 / 4}$, and $A_{\epsilon}^{(\text {ord })} / \sqrt{B_{\epsilon}}=-A_{\epsilon}^{(+)} / \sqrt{B_{\epsilon}}=1$.

${ }^{27}$ In the $d / 4$ Ising model $x_{\phi}, x_{\epsilon}$, and $C_{\phi \phi \epsilon} /\left(B_{\phi} B_{\epsilon}^{1 / 2}\right)$ attain their Gaussian values 1,2, and $-2^{1 / 2}$, respectively, while $A_{\phi}^{(+)} / \sqrt{B_{\phi}}=6 \varepsilon^{-1 / 2}$, $A_{\epsilon}^{(+)} / \sqrt{B_{\epsilon}}=-2^{3 / 2} 9 / \varepsilon, \quad C^{(+)}=4 \pi^{2} \varepsilon / 45, \quad X_{\phi, \phi}^{(+)}(y) \quad$ equals $y^{2} /(y+1)+12[1$ $\left.-\left(y^{-1}+(1 / 2)\right) \ln (y+1)\right]$, and $X_{\phi, \epsilon}^{(+)}(y)$ equals $-6 \sqrt{2 / \varepsilon} X_{\phi, \phi}^{(+)}(y)$, compare Refs. 8, 18, and 25.

${ }^{28}$ P. G. de Gennes, Scaling Concepts in Polymer Physics (Cornell University, Ithaca, 1979).

${ }^{29}$ T. W. Burkhardt, E. Eisenriegler, and I. Guim, Nucl. Phys. B 316, 559 (1989); T. W. Burkhardt and E. Eisenriegler, ibid. 424, 487 (1994).

${ }^{30}$ E. Eisenriegler and A. Bringer, J. Chem. Phys. 127, 034904 (2007).

${ }^{31}$ R. Tuinier, G. A. Vliegenthart, and H. N. W. Lekkerkerker, J. Chem. Phys. 113, 10768 (2000); R. Tuinier, G. A. Vliegenthart, and H. N. W.
Lekkerkerker, Macromolecules 34, 4636 (2001).

${ }^{32}$ Generally, for a polymer perturbation of finite extent, the densities $\mathcal{K}_{S, P}$ have a nontrivial limit for $\mathcal{R}_{g} \rightarrow \infty$, which is given by the critical ratios in Eqs. (3.1) and (3.2) in Ref. 30, while for an infinitely extended planar wall $P, \mathcal{K}_{S \text {,hs }}$ tends to zero.

${ }^{33}$ The amplitudes depend on the internal lengths of the small object and, like a multipole expansion, the expansion only includes operators that are consistent with all symmetries of the object. The usual operator product expansions and the small particle expansions for spherical (Refs. 17 and 18) or nonspherical (Ref. 25) particles are other expansions with these characteristics.

${ }^{34}$ Interesting examples are the density profiles of $\phi$ or $\epsilon$ induced by two circular disks in the critical $d=2$ Ising model.

${ }^{35}$ T. W. Burkhardt and I. Guim, Phys. Rev. B 47, 14306 (1993). 